\title{
21. GEOCHEMISTRY OF BASALTS FROM HOLES 519A, 520, 522B, AND 524, DEEP SEA DRILLING PROJECT LEG 73 (SOUTH ATLANTIC)1
}

\author{
V. J. Dietrich, Department of Earth Sciences, Eidgenössische Technische Hochschule Zürich, \\ $\mathrm{CH}-8092$ Zürich, Switzerland \\ M. F. Carman, Jr., Department of Geological Sciences, University of Houston, Houston, Texas \\ A. Wyttenbach, Eidgenössisches Reaktorinstitut, CH-5303 Würenlingen, Switzerland \\ and \\ E. H. McKee, U.S. Geological Survey, Menlo Park, California
}

\begin{abstract}
During Deep Sea Drilling Project Leg 73 (South Atlantic), basaltic pillow lava, flows, and sills were encountered in Holes 519A, 520, 522B, and 524. Paleomagnetic data indicate that the basalts from Holes 519A (magnetic Anomaly $5^{1}$ ) and 522B (Anomaly 16) have ages of $\sim 12$ m.y. and $\sim 38$ m.y., respectively.

The major- and trace- (including rare-earth-) element characteristics of the Hole 519A basalts (a total of $27 \mathrm{~m}$ ) demonstrate that these basalts are typical normal-type mid-ocean-ridge basalts (N-type MORB). In composition the basalts overlap olivine tholeiites from other normal Mid-Atlantic Ridge segments. Both the spectra of incompatible, or less-hygromagmatophile elements (such as $\mathrm{Ti}, \mathrm{V}, \mathrm{Y}$, and $\mathrm{Zr}$ ) and $\mathrm{REE}$ abundances indicate that these basalts are the result of a low-pressure fractionation of olivine, spinel, and plagioclase prior to eruption.

In Hole 520 only $1.7 \mathrm{~m}$ of basalt were recovered from a total drilling depth of $10.5 \mathrm{~m}$. These pillow basalts crystallized from fairly evolved (N-type MORB) tholeiitic melts.

In total, $19 \mathrm{~m}$ of basaltic pillow lavas and flows were penetrated in Hole 522B. Thirteen cooling units were distinguished on the basis of glassy margins and fine quench textures. In contrast to Holes 519A and 520, the basalts of the Hole 522B ridge section can be divided into two major groups of tholeiites: (1) Cooling Units 1 through 12 and (2) Cooling Unit 13. The basalts in this ridge section are also N-type MORBs but are generally more differentiated than those of Holes 519A and 520. The lowermost basalts (Cooling Unit 13) have the most primitive composition and make up a compositional group distinct from the more evolved basalts in the twelve units above it.

Hole 524 was drilled on the south flank of the Walvis Ridge and thus provided samples from a more complex part of the South Atlantic seafloor. Three different basaltic rock suites, interlayered with volcanic detrital sediments, were encountered. The rock suites are, from top to bottom, an alkali basaltic pillow lava; a 16-m-thick alkaline diabase sill with an age of $\sim 65 \mathrm{~m}$.y. (according to K-Ar dating and planktonic foraminifers); and a second sill that is approximately $9 \mathrm{~m}$ thick, $\sim 74 \mathrm{~m} . \mathrm{y}$. in age, and tholeiitic in composition, thus contrasting strongly with the overlying alkaline rocks.

The alkali basalts of Hole 524 show chemical characteristics that are very similar to the basaltic lavas of the Tristan da Cunha group volcanoes, which are located approximately $400 \mathrm{~km}$ east of the Mid-Atlantic Ridge crest. Thus, the Walvis Ridge may plausibly be interpreted as a line of hot-spot alkaline volcanoes.
\end{abstract}

\section{INTRODUCTION}

One purpose of the Leg 73 cruise was to define the nature of the poorly developed Mid-Atlantic Ridge (MAR) crest in the South Atlantic as well as to shed light on the nature and origin of the Walvis Ridge. Since Morgan (1972) and Wilson (1973) suggested that this aseismic ridge might have originated from hot-spot or mantle plume activity, it has been suspected of being composed of a chain of submarine volcanic seamounts (Goslin and Sibuet, 1975).

Alkaline volcanic rocks were subsequently dredged from different areas along the ridge (Hekinian, 1972; Humphris and Thompson, 1981) and drilled on DSDP Leg 39 in Hole 359 (Fodor et al., 1977).

During Leg 73 (South Atlantic), oceanic basement was encountered in Holes 519A, 520, 522B, and 524 (26-29 $9^{\circ} \mathrm{S}$ latitude and $11^{\circ} \mathrm{W}-3^{\circ} 30^{\prime} \mathrm{E}$ longitude; Fig. 1). In Holes 519A, 520, and 522B the igneous rocks proved

\footnotetext{
${ }^{1}$ Hsü, K. J., LaBrecque, J. L., et al., Init. Repts. DSDP, 73: Washington (U.S. Govt. Printing Office).
}

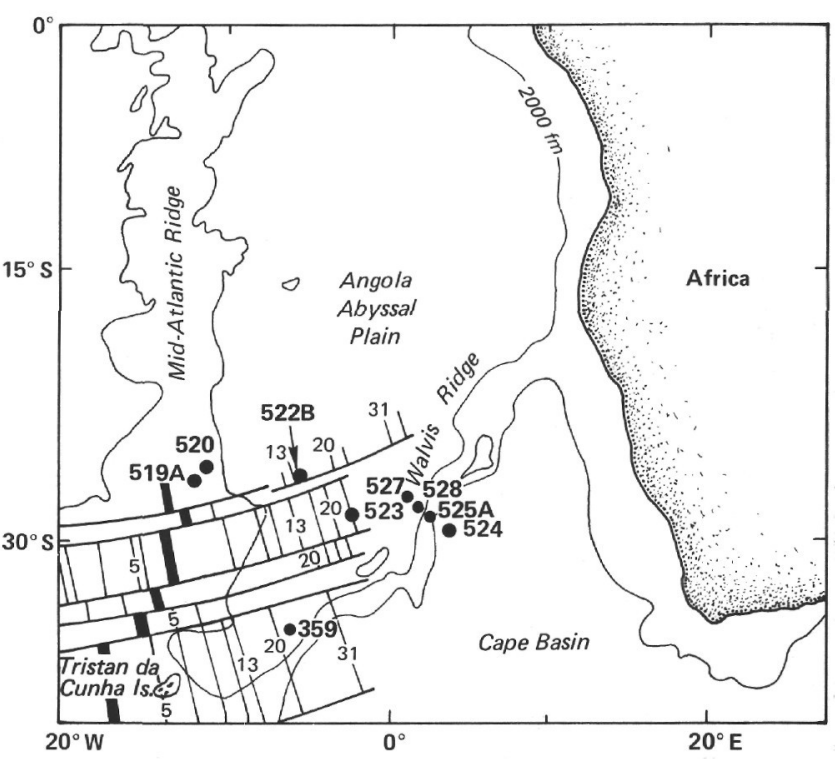

Figure 1. Southeast Atlantic with selected sites drilled during DSDP Legs 39,73 , and 74 . Smaller numbers denote marine magnetic anomalies. 
to be typical mid-ocean-ridge tholeiitic basalts (MORB), both chemically and petrographically. The rocks in Hole 524 , which was drilled on the southeastern flank of the Walvis Ridge, were more complex: alkalic pillows and a sill appeared in an upper sequence, and a slightly enriched tholeiitic basalt appeared in a lower multiple sill. Drilling on the Walvis Ridge continued on DSDP Leg 74 in Holes 525A, 527, and 528 (Richardson et al., 1982; Richardson et al., in press), yielding alkaline basalts very similar to those described here from Hole 524 .

This paper presents the major-element and trace-element compositions of the basalts in Holes 519A, 520, 522B, and 524. The major-element data are also represented in stratigraphic sequences (Figs. 2-4). Details of the stratigraphy of the cooling units are discussed in the site chapters and illustrated in the chapter by Carman et al. on the petrography and mineralogy of the basaltic rocks.

\section{ANALYTICAL TECHNIQUES}

Six samples of basalt were dated by the $\mathrm{K}-\mathrm{Ar}$ technique (Table 1) in the U.S. Geological Survey (USGS) laboratory in Menlo Park, California. The samples were prepared by being crushed, sieved (to 60100 mesh size), washed, and treated for $30 \mathrm{~min}$. in $14 \% \mathrm{HNO}_{3}$ and 1 min. in $5 \%$ HF solutions. The method used for gas extraction in the high-vacuum system is similar to that described by Dalrymple and Lanphere (1969). Argon analysis was performed by standard isotopedilution procedures and by using a $60^{\circ}$ sector, $15.2-\mathrm{cm}$-radius, Neirtype mass spectrometer. Potassium analyses were performed by a lithium metaborate flux fusion-flame photometry technique in which the lithium serves as an internal standard (Ingamells, 1970).
Major-element bulk chemical composition was determined by the $\mathrm{X}$-ray fluorescence (XRF) analysis (Tables 2-13) of glass beads; the glass beads were fused from ignited powders plus $\mathrm{Li}_{2} \mathrm{~B}_{4} \mathrm{O}_{7}(1 / 5$ ratio) in a gold-platinium pan at $1150^{\circ} \mathrm{C}$ (Dietrich et al., 1976). The XRF analyses were performed with an automated Philips sequential spectrometer (PW 1450) at the Eidgenössische Materialprüfungsanstalt, Dubendorf, Switzerland. The data were corrected for drift, background, and matrix effects. Twelve USGS reference rock samples were used for calibration. $\mathrm{FeO}$ content was determined colorimetrically. The analysis of $\mathrm{CO}_{2}$, which was performed with a Coulomat CS 701, was based on coulometric alkalimetric titration (Sixta, 1977). $\mathrm{H}_{2} \mathrm{O}^{+}$content was calculated from ignition loss, iron oxidation, and $\mathrm{CO}_{2}$. Nb, $\mathrm{Zr}, \mathrm{Y}, \mathrm{Sr}, \mathrm{U}, \mathrm{Rb}, \mathrm{Th}, \mathrm{Pb}, \mathrm{Ga}, \mathrm{Zn}, \mathrm{Cu}, \mathrm{Ni}, \mathrm{Co}, \mathrm{Cr}, \mathrm{V}, \mathrm{Ce}$, $\mathrm{Nd}, \mathrm{Ba}, \mathrm{La}, \mathrm{Sc}$, and $\mathrm{S}$ trace-element abundances were also analyzed by using X-ray fluorescence; $10-\mathrm{g}$ powder samples were analyzed by using the synthetic background method, in which major-element contents are known. A computer program was used to calculate background, interference, and mass absorption effects as well as standard deviations (Nisbet et al., 1979). The USGS reference samples were used for calibration. The resulting accuracies were \pm 2 to $3 \%$ at $1000 \mathrm{ppm}, \pm 5$ to $10 \%$ at $100 \mathrm{ppm}$, and \pm 10 to $20 \%$ at $10 \mathrm{ppm}$. A chromium tube was used, and detection limits were around $3 \mathrm{ppm}$ for most trace elements.

The rare-earth elements (REE; Table 3) were determined by radiochemical neutron activation (Bajo and Wyttenbach, 1980) on 100 -mg powder samples. The samples and standards were irradiated together in the Saphir reactor for a dose of $2 \cdot 10^{17}$ neutrons $/ \mathrm{cm}^{2}$. Afterwards the samples were twice taken to dryness with $\mathrm{HClO}_{4} / \mathrm{HF}$ and then treated in a Teflon-lined bomb with $\mathrm{HF} / \mathrm{HNO}_{3} / \mathrm{HCl}$ at $200^{\circ}$ for $16 \mathrm{hr}$. The resulting solution was twice taken to dryness with $\mathrm{HClO}_{4}$. The dissolved samples were extracted twice. The first extraction (with HTTA $/ \mathrm{CHCL}_{3}$ at $\mathrm{pH} 1.4$ ) removed ${ }^{46} \mathrm{Sc},{ }^{59} \mathrm{Fe}$, and most ${ }^{60} \mathrm{Co}$ and was discarded. The second extraction (with HTTA/phenanthroline/ $\mathrm{CHCL}_{3}$ at $\mathrm{pH}$ 2) removed all rare earths. Back-extraction with $\mathrm{HCl}$ yielded the rare earths with excellent radiochemical purity. The chemical yield of the dissolution and extraction steps was always checked with a ${ }^{139} \mathrm{Ce}-$

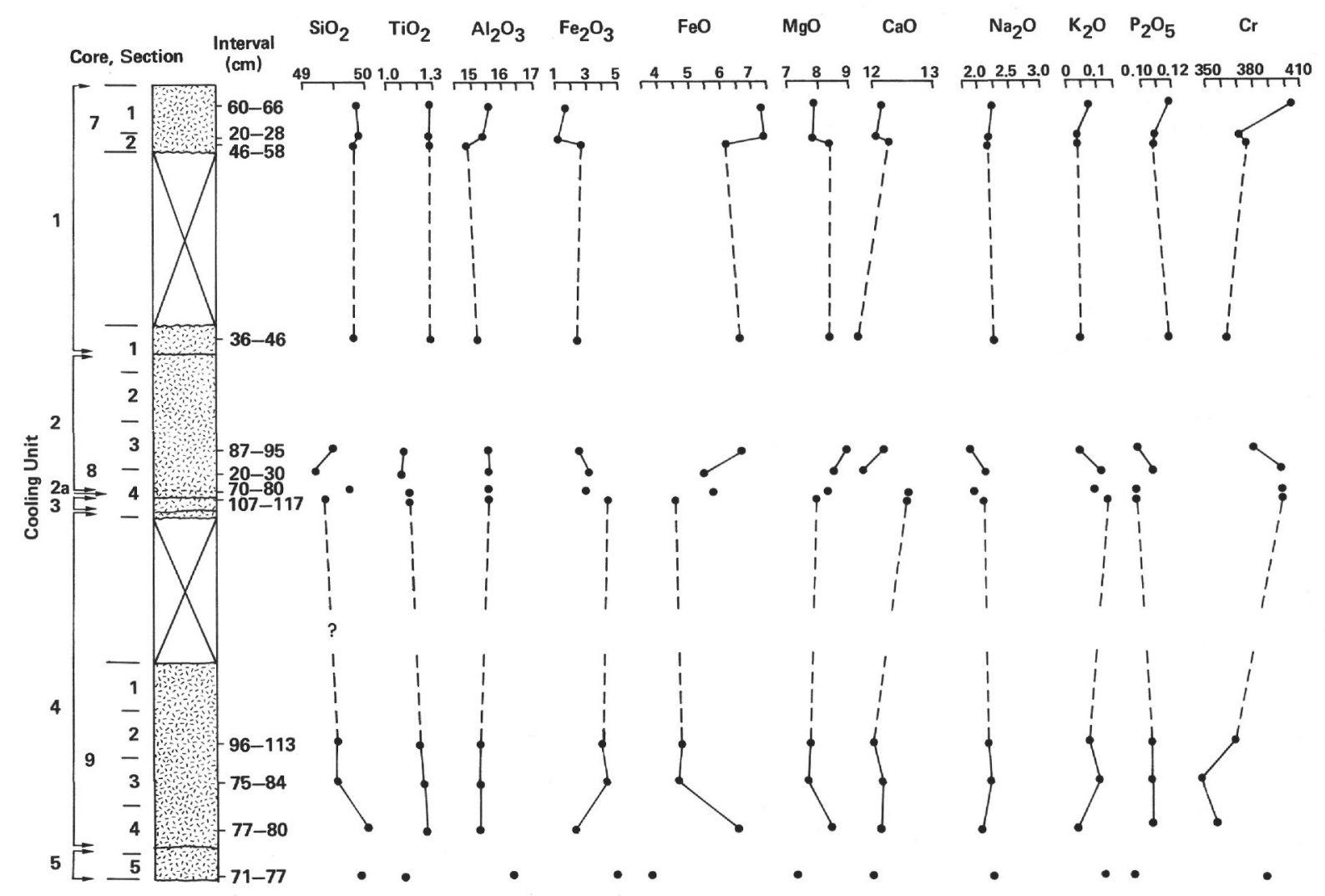

Figure 2. Downhole chemical variations in DSDP Hole 519A basalts. Chemical quantities are in wt.\% except for $\mathrm{Cr}$, which is in ppm. Cooling Units 1 through 5 consist of aphyric basalts (flows are indicated by an igneous pattern in the lithologic column). The upper part of Cooling Unit 1 is probably pillow lava. 


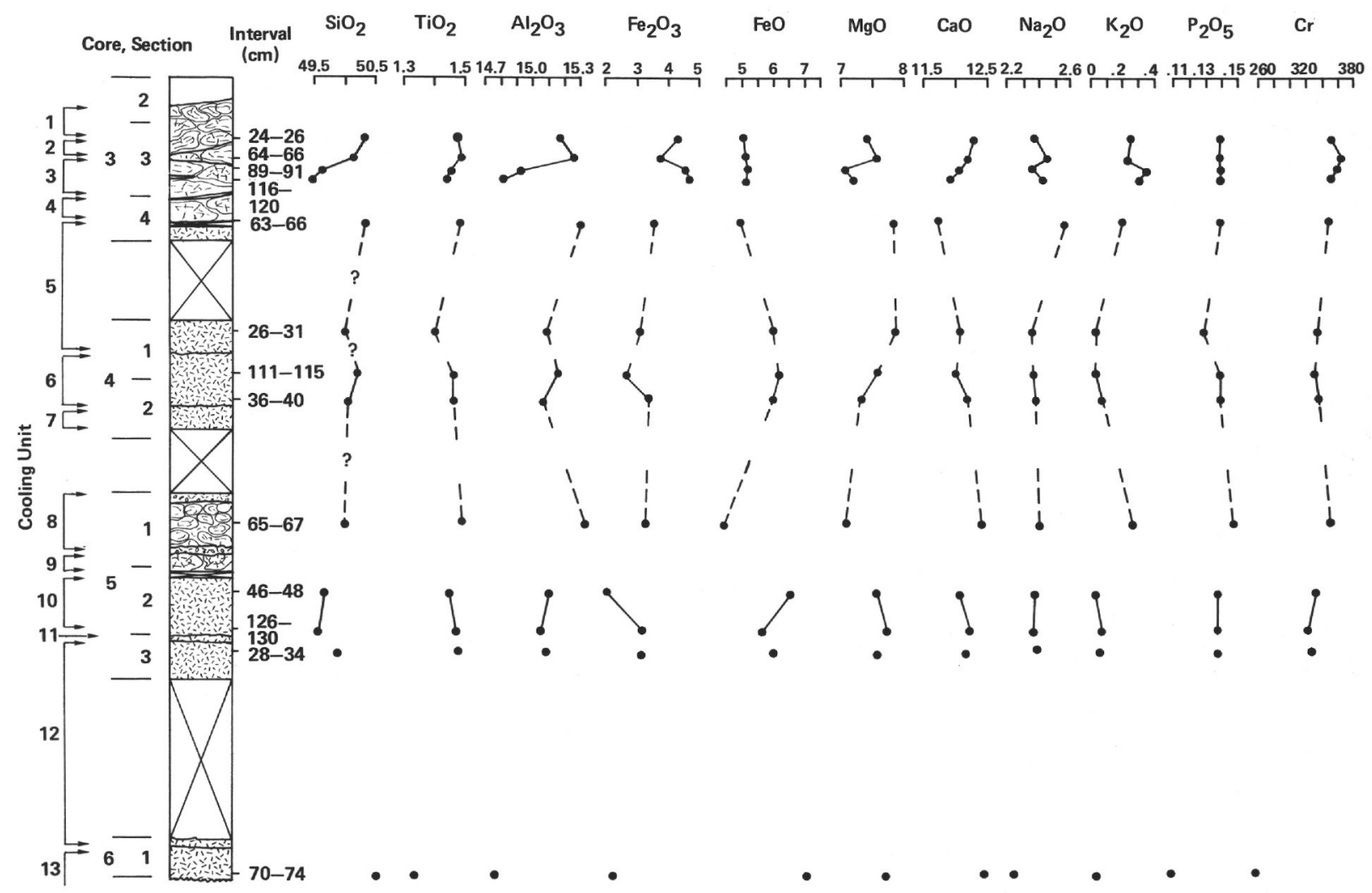

. Figure 3. Downhole chemical variations in Hole 522B basalts. Chemical quantities as in Fig. 2. Lithologic column: igneous pattern $=$ flows, otherwise pillow lava.

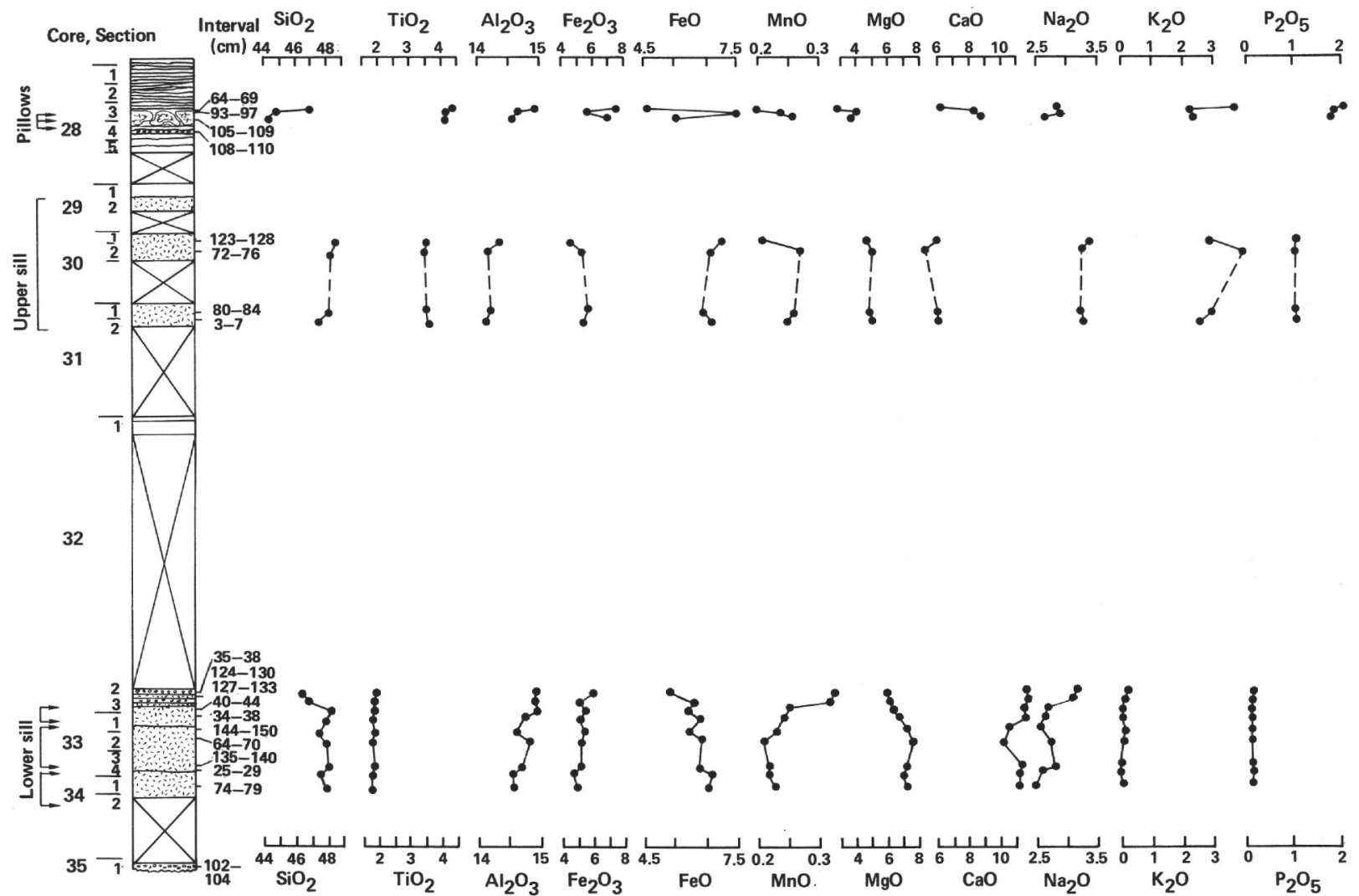

Figure 4. Downhole chemical variations in Hole 524 basalts. Chemical quantities are in wt.\%. Lithologic column: igneous pattern $=$ sills, in the upper part a pillow lava; horizontal lines with open circles = volcanic detrital sediments. 
Table 1. Analytical data of K-Ar age determinations of Leg 73 basalts.

\begin{tabular}{|c|c|c|c|c|c|}
\hline $\begin{array}{c}\text { Sample } \\
\text { (interval in } \mathrm{cm} \text { ) }\end{array}$ & $\begin{array}{l}\text { Cooling } \\
\text { Unit }\end{array}$ & $\begin{array}{c}\mathrm{K}_{2} \mathrm{O} \\
\text { (wt.\%) }\end{array}$ & $\begin{array}{l}{ }^{40} \mathrm{Ar} * \mathrm{a} \\
\text { (rad. }{ }^{40} \mathrm{Ar} \text { ) }\end{array}$ & $\begin{array}{c}{ }^{40} \mathrm{Ar} * \mathrm{a} \\
(\%)\end{array}$ & $\underset{\text { (m.y.) }}{\text { Age }}$ \\
\hline 519A-8-1, 26-37 (Piece 1B) & 1 & 0.039 & $4.86754 \times 10^{-12}$ & 2.4 & $84.7 \pm 20$ \\
\hline 519A-9-4, 41-47 (Piece 3C) & 4 & 0.028 & $4.79255 \times 10^{-12}$ & 1.7 & $115.1 \pm 50$ \\
\hline 522B-4-2, 2-9 (Piece 1A) & 6 & 0.040 & $2.93994 \times 10^{-11}$ & 1.2 & $50.3 \pm 43$ \\
\hline 522B-5-3, 7-11 (Piece 1B) & 11 & 0.039 & $3.3090 \times 10^{-12}$ & 10.0 & $58.0 \pm 7$ \\
\hline $524-31-1,75-80$ (Piece 8A) & Upper sill & 4.07 & $3.87278 \times 10^{-10}$ & 77 & $64.9 \pm 0.8$ \\
\hline 524-34-1, 79-83 (Piece 1D) & Lower sill & 0.189 & $2.04286 \times 10^{-11}$ & 59 & $73.6 \pm 1.6$ \\
\hline
\end{tabular}

spike and was usually found to be from 95 to $100 \%$. Samples and standards were counted as aqueous solutions with a volume of $15 \mathrm{ml}$. The $\gamma$-spectra were taken with a planar intrinsic Ge-detector (FWHM 700 $\mathrm{eV}$ at $100 \mathrm{keV}$ ) in the energy range up to $500 \mathrm{keV}$. Each sample was counted three times $(7,20,35$ days after irradiation) for 4 to $6 \mathrm{hr}$. Evaluation of the $\gamma$-spectra was done on a Ciber- 6000 with the JANE computer program (Schubiger et al., 1978).

Precision and accuracy were checked by repetitive analyses of BCR-1 $(n=6): \mathrm{La}=24.8 \pm 0.5, \mathrm{Ce}=53.3 \pm 1.0, \mathrm{Nd}=28.2 \pm 0.5$, $\mathrm{Sm}=6.8 \pm 0.14, \mathrm{Eu}=1.95 \pm 0.02, \mathrm{Gd}=6.8 \pm 0.2, \mathrm{~Tb}=0.9 \pm 0.03$, $\mathrm{Tm}=0.5 \pm 0.015, \mathrm{Yb}=3.4 \pm 0.07, \mathrm{Lu}=0.53 \pm 0.015$. Precision was determined entirely by counting statistics $(5 \%$ for Gd and $\mathrm{Tm}$, better than $3 \%$ for all others). Accuracy was estimated from a comparison of our results with the values (mostly from isotopic dilution analysis) given by Taylor and Gorton (1977). Deviations were 3\% or smaller for all elements except $\mathrm{Gd}(+5 \%)$ and $\mathrm{Tb}(-8 \%)$.

\section{DATING}

$\mathrm{K}$-Ar data were obtained on two samples each from Holes 519A, 522B, and 524. As shown in Table 1, two of the samples (those from Hole 524) yielded ages that were reasonable compared with paleontologic and paleomagnetic criteria; the other four samples (from Holes 519A and 522B) contained such large relative amounts of atmospheric ${ }^{40} \mathrm{Ar}$ contaminant that the calculated age could not be reasonably evaluated because of the large spread of analytical precision $( \pm)$. However, the paleomagnetic results from the basalts of Holes 519A and 522B placed them in Magnetic Anomalies $5^{1}$ and 16, respectively, with corresponding ages of about $12 \mathrm{~m} . \mathrm{y}$. and $38 \mathrm{~m} . \mathrm{y}$.

In these holes the correlation between the biostratigraphic zones and the magnetostratigraphic column was good. The basalts of Hole 524 were tentatively correlated with Anomaly 31, indicating an age of about $69 \mathrm{~m} . \mathrm{y}$. According to the planktonic foraminifers found in the volcanic detrital sediments in Cores 26 and 35 (see the site chapters), all the basaltic rocks drilled in Hole 524 are of Upper Cretaceous age, possibly Maestrichtian to late Maestrichtian.

Since the two radiometrically dated samples come from units interpreted as sills from contact relations and texture, their magnetic age relations with the enclosing sediments could well be anomalous. In fact, the radiometric age of the alkalic upper sill correlates better with Anomaly 27 or 28 than with 31 . The tholeiitic lower sill is seen to be distinctly older than Anomaly 31 . Its age apparently relates it to the second magnetic reversal period preceding Anomaly 31. This age is plausible, in

Table 2. Major- and trace-element abundances of basalts from Hole 519A.

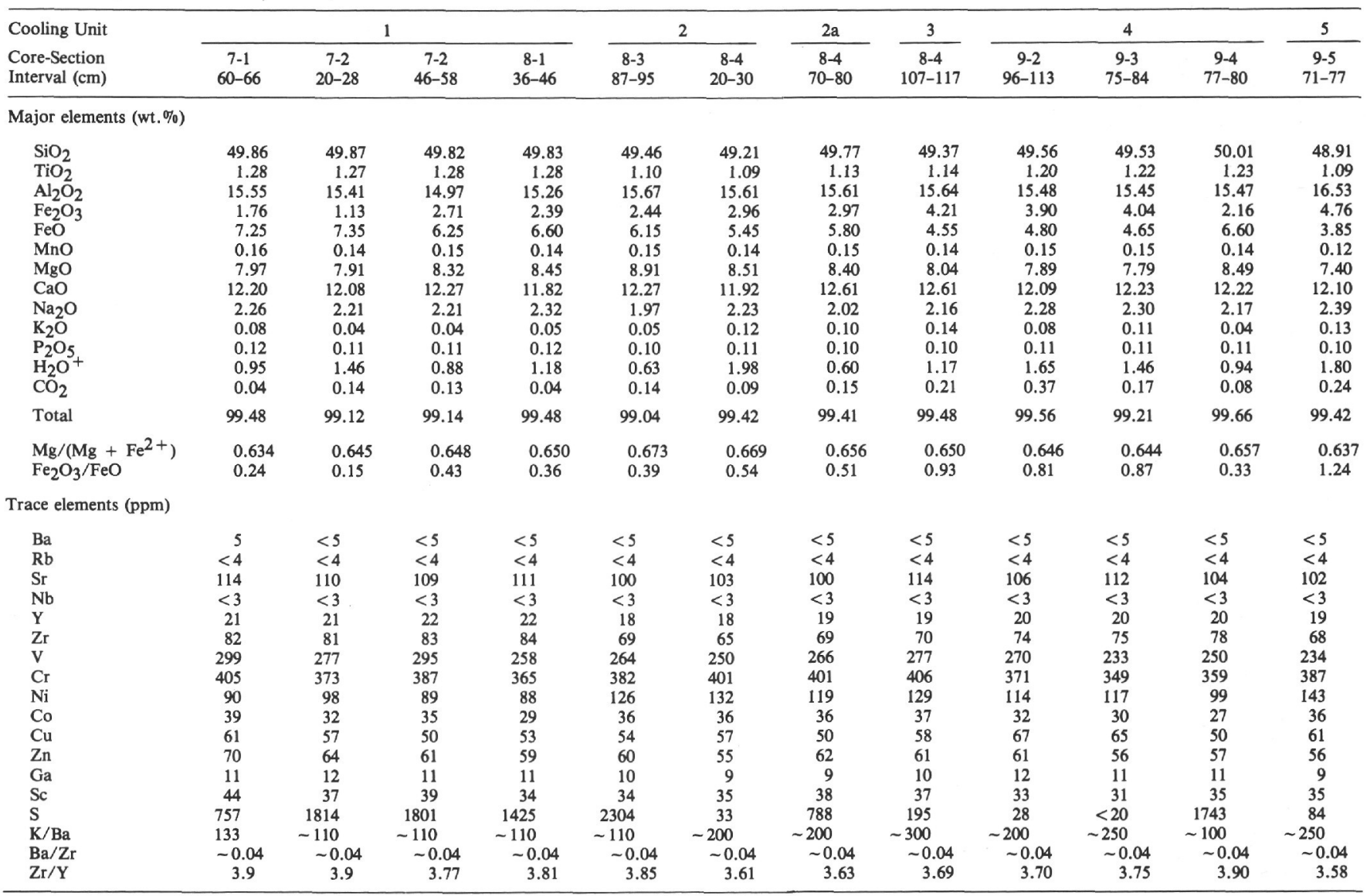


Table 3. Rare-earth-element abundances (ppm) of basalts from Holes 519A, 520, 522B, and 524.

\begin{tabular}{|c|c|c|c|c|c|c|c|c|c|c|c|c|c|}
\hline \multirow{3}{*}{$\begin{array}{l}\text { Hole } \\
\text { Core-Section } \\
\text { Interval }(\mathrm{cm})\end{array}$} & \multicolumn{3}{|c|}{ 519A } & \multirow{3}{*}{$\frac{520}{31-1}$} & \multicolumn{4}{|c|}{$522 \mathrm{~B}$} & \multicolumn{3}{|c|}{524 (upper part) } & \multicolumn{2}{|c|}{524 (lower part) } \\
\hline & $7-2$ & $8-3$ & $9-4$ & & $3-4$ & $4-2$ & $5-2$ & $6-1$ & $28-3$ & $30-2$ & $31-2$ & $32-3$ & $34-1$ \\
\hline & $46-58$ & $87-95$ & $77-80$ & & $64-66$ & $36-40$ & $126-130$ & $70-74$ & $93-97$ & $72-76$ & $3-7$ & $40-44$ & $74-79$ \\
\hline $\mathrm{La}$ & 2.32 & 2.04 & 2.16 & 4.29 & 3.47 & 3.59 & 3.36 & 2.36 & 62.6 & 53.9 & 53.9 & 6.92 & 6.96 \\
\hline $\mathrm{Ce}$ & 7.97 & 6.63 & 7.29 & 14.6 & 10.45 & 10.17 & 10.43 & 7.88 & 131.9 & 109.9 & 111.5 & 18.6 & 17.6 \\
\hline Nd & 7.89 & 6.62 & 7.33 & 12.9 & 9.58 & 8.70 & 9.32 & 8.40 & $67^{a}$ & $52^{\mathrm{a}}$ & $54^{\mathrm{a}}$ & 14.8 & 14.4 \\
\hline $\mathrm{Sm}$ & 2.70 & 2.30 & 2.61 & 4.62 & 3.25 & 3.04 & 3.12 & 2.78 & 15.3 & 12.0 & 12.3 & 5.28 & 5.09 \\
\hline Eu & 0.98 & 0.86 & 0.96 & 1.49 & 1.18 & 1.08 & 1.11 & 1.08 & 5.95 & 4.6 & 4.78 & 1.77 & 1.67 \\
\hline $\mathrm{Gd}$ & 3.74 & 3.04 & 3.74 & 6.11 & 4.35 & 4.18 & 4.39 & 4.22 & n.d. & n.d. & n.d. & 5.93 & 5.83 \\
\hline $\mathrm{Tb}$ & 0.59 & 0.52 & 0.59 & 1.02 & 0.77 & 0.69 & 0.70 & 0.70 & 1.71 & 1.53 & 1.50 & 1.08 & 1.00 \\
\hline $\mathrm{Tm}$ & 0.41 & 0.36 & 0.37 & 0.66 & 0.47 & 0.44 & 0.46 & $0.50^{\mathrm{b}}$ & n.d. & n.d. & n.d. & 0.60 & 0.55 \\
\hline $\mathrm{Yb}$ & 2.59 & 2.28 & 2.54 & 4.27 & 3.05 & 2.93 & 2.92 & 3.07 & 2.98 & 3.00 & 3.04 & 4.06 & 3.82 \\
\hline $\mathrm{Lu}$ & 0.407 & 0.36 & 0.39 & 0.68 & 0.48 & 0.46 & 0.45 & 0.47 & 0.46 & 0.46 & 0.47 & 0.65 & 0.62 \\
\hline$(\mathrm{Ce} / \mathrm{Sm})_{\mathrm{N}}$ & 0.70 & 0.68 & 0.66 & 0.75 & 0.76 & 0.79 & 0.79 & 0.67 & 2.03 & 2.17 & 2.14 & 0.83 & 0.82 \\
\hline $\mathrm{Ce} / \mathrm{Yb}$ & 3.08 & 2.91 & 2.87 & 3.42 & 3.43 & 3.47 & 3.57 & 2.57 & 44.3 & 36.6 & 36.7 & 4.58 & 4.61 \\
\hline $\mathrm{Ce} / \mathrm{Zr}$ & 0.096 & 0.096 & 0.093 & 0.095 & 0.112 & 0.108 & 0.111 & 0.096 & 0.517 & 0.383 & 0.405 & 0.128 & 0.131 \\
\hline $\mathrm{Ba} / \mathrm{La}$ & $1-2.5$ & $1-2.5$ & $1-2.5$ & 2.6 & 2.6 & 2.5 & 2.7 & 2.97 & 21.3 & 20.2 & 19.1 & 9.7 & 8.8 \\
\hline
\end{tabular}

Note: n.d. = not determined.

a Determined with X-ray fluorescence.

b Large error due to interference.

Table 4. Normative mineralogy (CIPW) of basalts from Hole 519A.

\begin{tabular}{|c|c|c|c|c|c|c|c|c|c|c|c|c|}
\hline \multirow{2}{*}{$\begin{array}{l}\text { Cooling Unit } \\
\text { Core-Section } \\
\text { Interval }(\mathrm{cm})\end{array}$} & \multicolumn{4}{|c|}{1} & \multicolumn{2}{|c|}{2} & \multirow{2}{*}{ 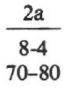 } & \multirow{2}{*}{$\frac{3}{\frac{8-4}{107-117}}$} & \multicolumn{4}{|c|}{4} \\
\hline & $\begin{array}{c}7-1 \\
60-66\end{array}$ & $\begin{array}{c}7-2 \\
20-28\end{array}$ & $\begin{array}{c}7-2 \\
46-58\end{array}$ & $\begin{array}{c}8-1 \\
36-46\end{array}$ & $\begin{array}{c}8-3 \\
87-95\end{array}$ & $\begin{array}{c}8-4 \\
20-30\end{array}$ & & & $\begin{array}{c}9-2 \\
96-113\end{array}$ & $\begin{array}{c}9-3 \\
75-84\end{array}$ & $\begin{array}{c}9-4 \\
77-80\end{array}$ & $\begin{array}{c}9-5 \\
71-77\end{array}$ \\
\hline qz & 0.00 & 0.33 & 0.00 & 0.00 & 0.00 & 0.00 & 0.00 & 0.00 & 0.00 & 0.00 & 0.00 & 0.00 \\
\hline or & 0.48 & 0.24 & 0.24 & 0.30 & 0.30 & 0.73 & 0.60 & 0.85 & 0.49 & 0.67 & 0.24 & 0.79 \\
\hline$a b$ & 19.41 & 19.20 & 19.08 & 19.98 & 16.98 & 19.39 & 17.35 & 18.67 & 19.86 & 19.97 & 18.62 & 20.82 \\
\hline an & 32.54 & 32.86 & 31.43 & 31.62 & 34.40 & 33.12 & 33.72 & 33.26 & 32.70 & 32.34 & 32.81 & 34.99 \\
\hline (wo & 11.63 & 11.28 & 12.15 & 11.27 & 10.87 & 10.99 & 11.75 & 11.95 & 10.81 & 11.73 & 11.45 & 10.26 \\
\hline $\operatorname{di}\{$ en & 6.84 & 6.75 & 7.29 & 6.78 & 6.70 & 6.74 & 7.08 & 7.15 & 6.46 & 6.99 & 6.94 & 6.03 \\
\hline$l_{\mathrm{fs}}$ & 4.22 & 3.94 & 4.22 & 3.89 & 3.55 & 3.62 & 4.03 & 4.17 & 3.79 & 4.13 & 3.89 & 3.72 \\
\hline jen & 11.42 & 13.48 & 12.61 & 12.02 & 13.65 & 11.28 & 12.61 & 10.60 & 13.36 & 11.45 & 13.01 & 9.34 \\
\hline${ }^{n y}\left\{\begin{array}{l}\text { fs } \\
\text {. }\end{array}\right.$ & 7.05 & 7.87 & 7.31 & 6.90 & 7.23 & 6.06 & 7.18 & 6.18 & 7.84 & 6.77 & 7.29 & 5.77 \\
\hline fo & 1.33 & 0.00 & 0.87 & 1.84 & 1.59 & 2.64 & 1.08 & 1.89 & 0.30 & 1.03 & 1.05 & 2.53 \\
\hline$\}_{\mathrm{fa}}$ & 0.91 & 0.00 & 0.56 & 1.17 & 0.93 & 1.56 & 0.68 & 1.22 & 0.19 & 0.67 & 0.65 & 1.72 \\
\hline $\mathrm{mt}$ & 1.18 & 1.13 & 1.17 & 1.17 & 1.12 & 1.10 & 1.13 & 1.12 & 1.13 & 1.12 & 1.14 & 1.10 \\
\hline il & 2.47 & 2.48 & 2.48 & 2.47 & 2.13 & 2.13 & 2.18 & 2.21 & 2.35 & 2.38 & 2.37 & 2.13 \\
\hline ap & 0.29 & 0.27 & 0.27 & 0.29 & 0.24 & 0.27 & 0.24 & 0.24 & 0.27 & 0.27 & 0.26 & 0.24 \\
\hline Total & 99.77 & 99.84 & 99.67 & 99.71 & 99.69 & 99.64 & 99.64 & 99.51 & 99.54 & 99.52 & 99.73 & 99.45 \\
\hline $100 \mathrm{Mg} / \mathrm{Mg}+\mathrm{Fe}^{2+}$ & 63.43 & 64.54 & 64.82 & 65.05 & 67.25 & 66.87 & 65.60 & 65.00 & 64.61 & 64.39 & 66.68 & 63.72 \\
\hline D.I. ${ }^{a}$ & 19.89 & 19.78 & 19.32 & 20.28 & 17.28 & 20.12 & 17.95 & 19.51 & 20.35 & 20.64 & 18.86 & 21.61 \\
\hline pl (an) & 62.63 & 63.12 & 62.23 & 61.29 & 66.95 & 63.07 & 66.03 & 64.05 & 62.22 & 61.82 & 63.79 & 62.70 \\
\hline or & 0.92 & 0.46 & 0.48 & 0.58 & 0.58 & 1.37 & 1.16 & 1.60 & 0.92 & 1.26 & 0.46 & 1.40 \\
\hline$a b$ & 37.03 & 36.71 & 37.59 & 38.49 & 32.86 & 36.42 & 33.57 & 35.37 & 37.44 & 37.70 & 36.04 & 36.78 \\
\hline an & 62.06 & 62.83 & 61.93 & 60.93 & 66.56 & 62.21 & 65.27 & 63.03 & 61.65 & 61.04 & 63.50 & 61.82 \\
\hline
\end{tabular}

a Differentiation index (Thornton and Tuttle, 1960).

view of the rather large gap in core recovery between Cores 30 and $33 \mathrm{~m}$ (Fig. 4).

The results from Hole 524 demonstrate that between about 65 and 70 m.y. ago there was a fundamental change in the nature of seafloor volcanism from tholeiitic to alkalic in the region of the Walvis Ridge. The alkalic type of volcanism has continued to the present, giving rise to such volcanoes as those in the Tristan da Cunha group (Dunne, 1941; and Baker et al., 1964).

\section{CHEMISTRY}

\section{Hole 519A}

\section{Discussion}

The results of the analyses of the Hole 519A basalts (a total of $27 \mathrm{~m}$ of basalt) are given in Tables 2, 3, and
4. In major-element, trace-element, and rare-earth-element contents, the basalts are comparable to normaltype (N-type) mid-ocean-ridge basalts (MORB; Sun et al., 1979; Wood, Joron, et al., 1979; and Wood et al., 1980).

The basalts are slightly olivine normative (Table 4) except for Sample 519A-7-2, 20-28 cm in Cooling Unit 1 , which is quartz normative and falls into the right side of the MORB field (Thompson et al., 1972) of the basaltic tetrahedron (Fig. 5). In the ternary plagioclasepyroxene-olivine system (Fig. 6), the basalts cluster around the upper region of the olivine-plagioclase-melt cotectic line, where a distinction between olivine tholeiites and plagioclase tholeiites is very difficult. In the upper parts of Cooling Units 1, 2, and 4, the existence of plagioclase tholeiites seems to be obvious. 
Table 5. Major- and trace-element abundances of basalts from Hole 520

\begin{tabular}{|c|c|c|c|}
\hline $\begin{array}{l}\text { Core-Section } \\
\text { Interval }(\mathrm{cm})\end{array}$ & $\begin{array}{c}30-1 \\
101-110\end{array}$ & $\begin{array}{l}31-1 \\
7-10\end{array}$ & $\begin{array}{c}31-1 \\
16-22\end{array}$ \\
\hline \multicolumn{4}{|l|}{ Major elements (wt.\%) } \\
\hline $\begin{array}{l}\mathrm{SiO}_{2} \\
\mathrm{TiO}_{2} \\
\mathrm{Al}_{2} \mathrm{O}_{3} \\
\mathrm{Fe}_{2} \mathrm{O}_{3} \\
\mathrm{FeO} \\
\mathrm{MnO} \\
\mathrm{MgO} \\
\mathrm{CaO} \\
\mathrm{Na}_{2} \mathrm{O} \\
\mathrm{K}_{2} \mathrm{O} \\
\mathrm{P}_{2} \mathrm{O}_{5} \\
\mathrm{H}_{2} \mathrm{O}^{+} \\
\mathrm{CO}_{2}\end{array}$ & $\begin{array}{r}49.56 \\
2.11 \\
14.34 \\
5.28 \\
6.20 \\
0.18 \\
6.93 \\
10.74 \\
2.41 \\
0.39 \\
0.21 \\
1.02 \\
0.00\end{array}$ & $\begin{array}{r}49.61 \\
2.18 \\
14.69 \\
7.34 \\
3.95 \\
0.18 \\
6.40 \\
11.09 \\
2.55 \\
0.29 \\
0.21 \\
1.16 \\
0.00\end{array}$ & $\begin{array}{r}49.89 \\
2.11 \\
14.35 \\
3.51 \\
7.90 \\
0.19 \\
7.30 \\
10.63 \\
2.38 \\
0.10 \\
0.20 \\
0.85 \\
0.05\end{array}$ \\
\hline Total & 99.37 & 99.65 & 99.46 \\
\hline $\begin{array}{l}\mathrm{Mg} /\left(\mathrm{Mg}+\mathrm{Fe}^{2+}\right) \\
\mathrm{Fe}_{2} \mathrm{O}_{3} / \mathrm{FeO}\end{array}$ & $\begin{array}{l}0.549 \\
0.85\end{array}$ & $\begin{array}{l}0.538 \\
1.83\end{array}$ & $\begin{array}{l}0.560 \\
0.44\end{array}$ \\
\hline
\end{tabular}

Trace elements (ppm)

\begin{tabular}{lrrr}
$\mathrm{Ba}$ & 7 & 7 & 11 \\
$\mathrm{Rb}$ & $<4$ & $<4$ & $<4$ \\
$\mathrm{Sr}$ & 109 & 118 & 104 \\
$\mathrm{Nb}$ & $<3$ & $<3$ & $<3$ \\
$\mathrm{Y}$ & 44 & 44 & 42 \\
$\mathrm{Zr}$ & 156 & 158 & 153 \\
$\mathrm{~V}$ & 400 & 410 & 438 \\
$\mathrm{Cr}$ & 233 & 232 & 230 \\
$\mathrm{Ni}$ & 101 & 115 & 101 \\
$\mathrm{Co}$ & 50 & 52 & 44 \\
$\mathrm{Cu}$ & 37 & 41 & 41 \\
$\mathrm{Zn}$ & 94 & 97 & 97 \\
$\mathrm{Ga}$ & 12 & 14 & 14 \\
$\mathrm{Sc}$ & 46 & 43 & 48 \\
$\mathrm{~S}$ & 772 & 553 & 1121 \\
$\mathrm{~K} / \mathrm{Ba}$ & 463 & 344 & 75.5 \\
$\mathrm{Ba} / \mathrm{Zr}$ & 0.045 & 0.044 & 0.072 \\
$\mathrm{Zr} / \mathrm{Y}$ & 3.55 & 3.60 & 3.64 \\
\hline
\end{tabular}

Before basalt chemistry can be discussed, the effects of alteration have to be checked. Studies by Pearce and Cann (1973), Frey et al. (1974), Humphris and Thompson (1978), Floyd and Tarney (1979), Pearce and Norry (1973), and Staudigel et al. (1981) have provided useful data on the effects of low-temperature alteration on selected major, trace, and rare-earth elements. The tight grouping of some crucial mobile elements, such as $\mathrm{Na}_{2} \mathrm{O}$, $\mathrm{K}_{2} \mathrm{O}$, and $\mathrm{CaO}$ (Figs. 7 and $8 \mathrm{~A}$ ), the transition elements (Fig. 8B), and the hygromagmatophile (HYG) or incompatible elements $\mathrm{Ba}, \mathrm{Rb}$, and $\mathrm{Sr}$ indicate that the mobilization of these elements within the Hole 519 basalts is rather small. Nevertheless, most of the samples are slightly altered. This can be judged on the basis of the $\mathrm{Fe}_{2} \mathrm{O}_{3} / \mathrm{FeO}$ ratios $>0.15$ and on the $\mathrm{CO}_{2}$ and $\mathrm{H}_{2} \mathrm{O}^{+}$ contents (Table 2).

Typical for normal MORB tholeiites are the low amounts of the more-hygromagmatophile elements (Treuil and Varet 1973; and Wood, Joron, et al., 1979). These are elements such as $\mathrm{K}\left(\mathrm{K}_{2} \mathrm{O}=0.04-0.14\right.$ wt. \%; Figs. 7 and $8 \mathrm{~A}), \mathrm{Rb}(<4 \mathrm{ppm}), \mathrm{Ba}(\leq 5 \mathrm{ppm}$; Fig. $8 \mathrm{C})$, $\mathrm{Nb}(<3 \mathrm{ppm}), \mathrm{La}(2.5-3 \mathrm{ppm})$, and Ce (7.5-9 ppm),
Table 6. Normative mineralogy (CIPW) of basalts from Hole 520.

\begin{tabular}{|c|c|c|c|}
\hline $\begin{array}{l}\text { Core-Section } \\
\text { Interval }(\mathrm{cm})\end{array}$ & $\begin{array}{c}30-1 \\
101-110\end{array}$ & $\begin{array}{l}31-1 \\
7-10\end{array}$ & $\begin{array}{c}31-1 \\
16-22\end{array}$ \\
\hline $\mathrm{qz}$ & 0.13 & 0.38 & 1.07 \\
\hline or & 2.34 & 1.74 & 0.60 \\
\hline$a b$ & 20.73 & 21.90 & 20.44 \\
\hline an & 27.60 & 28.20 & 28.60 \\
\hline wo & 10.50 & 10.96 & 9.72 \\
\hline di $\{$ en & 5.57 & 5.75 & 5.23 \\
\hline$\left.\right|_{\mathrm{fs}}$ & 4.61 & 4.89 & 4.17 \\
\hline $\int_{y}^{\text {en }}$ & 11.98 & 10.43 & 13.23 \\
\hline hy $\left\{_{\text {fs }}\right.$ & 9.93 & 8.87 & 10.54 \\
\hline fo & 0.00 & 0.00 & 0.00 \\
\hline${ }^{o l}\left\{_{f a}\right.$ & 0.00 & 0.00 & 0.00 \\
\hline $\mathrm{mt}$ & 1.47 & 1.41 & 1.48 \\
\hline il & 4.07 & 4.20 & 4.07 \\
\hline ap & 0.51 & 0.50 & 0.48 \\
\hline Total & 99.44 & 99.23 & 99.62 \\
\hline $100 \mathrm{Mg} / \mathrm{Mg}+\mathrm{Fe}^{2+}$ & 54.94 & 53.86 & 55.96 \\
\hline D.I. ${ }^{a}$ & 23.20 & 24.02 & 22.11 \\
\hline pl(an) & 57.11 & 56.28 & 58.32 \\
\hline or & 4.62 & 3.36 & 1.21 \\
\hline$a b$ & 40.90 & 42.25 & 41.18 \\
\hline an & 54.47 & 54.40 & 57.61 \\
\hline
\end{tabular}

which are strongly partitioned into a fluid phase that yields distribution coefficients $D \leq 0.01$. The elements are depleted in the more-HYG elements relative to chondrites (Schilling, 1971; Frey et al., 1974; and Wood, Joron, et al., 1979), which is shown by the $\mathrm{Ba} / \mathrm{Zr}$ ratio of approximately 0.04 in Table 2 . The abundances of the transition metals $\mathrm{Co}, \mathrm{Ni}, \mathrm{Cr}, \mathrm{V}$, and $\mathrm{Sc}$ are shown in Figure 8B. They are also in the range of normal MORBs (Sun et al., 1979).

In general, the Hole 519A basalts overlap in composition the primitive olivine tholeiites from normal MidAtlantic Ridge segments, such as those from 60 to $55^{\circ} \mathrm{N}$ (Schilling, 1973; White and Schilling 1978) and 30 to $28^{\circ} \mathrm{N}$ (White and Schilling, 1978; and Sun et al., 1979).

On the basis of the less-hygromagmatophile or incompatible elements with $\mathrm{D} \leq 0.1$ (Figs. 8A and B), such as $\mathrm{Ti}\left(\mathrm{TiO}_{2}=1.09-1.28\right.$ wt. \%), $\mathrm{Zr}(65-84 \mathrm{ppm})$, and $\mathrm{Y}\left(18-22^{\circ} \mathrm{ppm}\right)$, as well as on the wide spread of $\mathrm{Ni}$ contents (88-143 ppm), the flow units of Hole 519A can be unambiguously separated and their eruption history discussed.

The lowermost flow, Cooling Unit 5 , has low $\mathrm{Ti}\left(\mathrm{TiO}_{2}\right.$ $=1.09$ wt. \%), $\mathrm{Zr}(68 \mathrm{ppm})$, and Y (19 ppm) contents for its particular $\mathrm{Mg}$ number of 0.637 and may therefore have originated from a different magma batch than the other cooling units (Figs. 9A and B). Also, the more highly altered nature of this flow suggests that it is older than the others. This is expressed by the $\mathrm{Fe}_{2} \mathrm{O}_{3} / \mathrm{FeO}$ ratio of 1.24 and the high amounts of $\mathrm{H}_{2} \mathrm{O}^{+}$and $\mathrm{CO}_{2}$. On the other hand, the high $\mathrm{Al}_{2} \mathrm{O}_{3}$ contents of 16.53 wt. $\%$ together with high $\mathrm{Cr}$ (387 ppm) and $\mathrm{Ni}(143 \mathrm{ppm})$ values can only be accomplished by a mixing process of 
Table 7. Major- and trace-element abundances of basalts from Hole 522B.

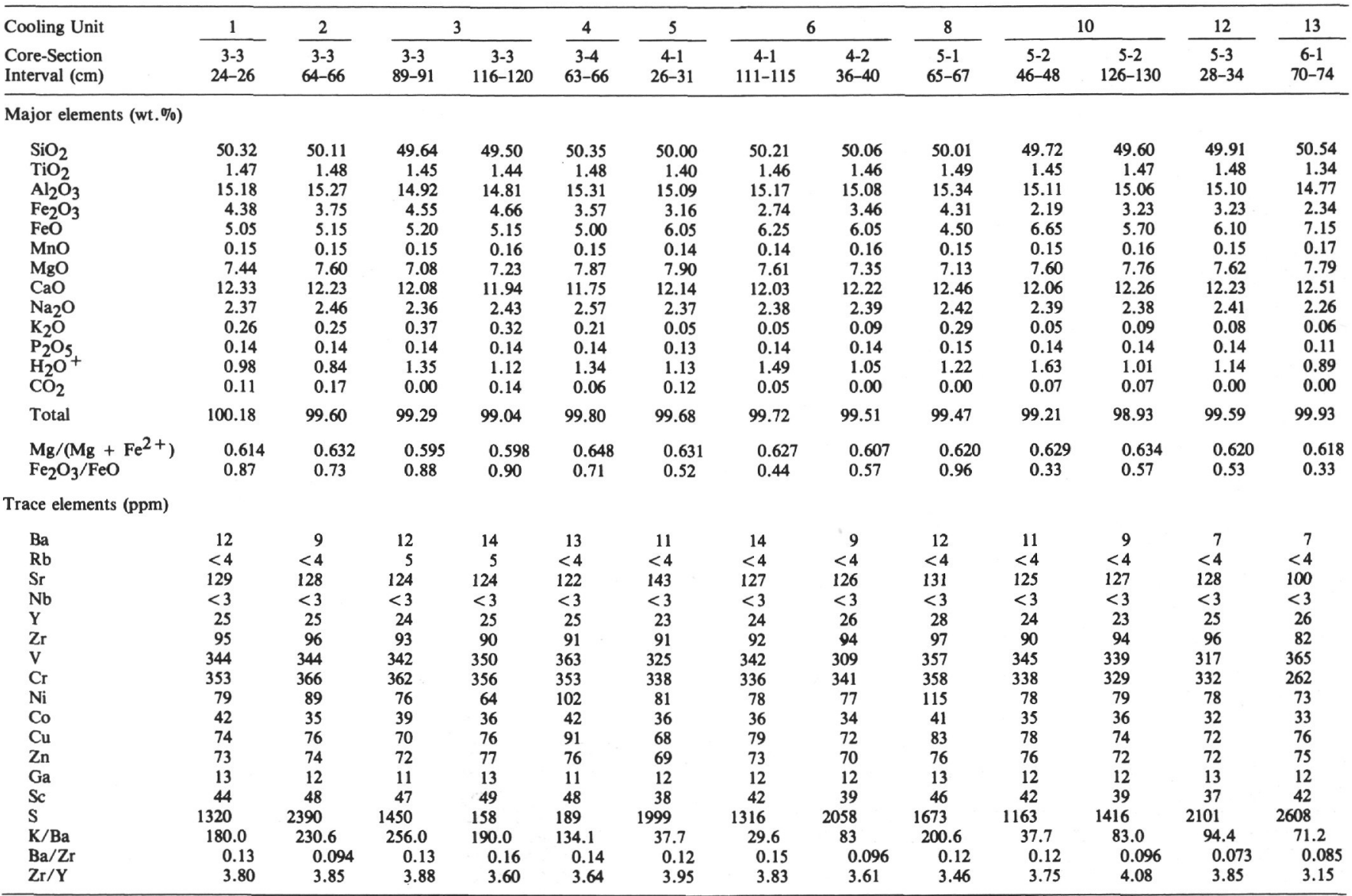

Table 8. Normative mineralogy (CIPW) of basalts from Hole 522B.

\begin{tabular}{|c|c|c|c|c|c|c|c|c|c|c|c|c|c|}
\hline \multirow{2}{*}{$\begin{array}{l}\text { Cooling Unit } \\
\text { Core-Section } \\
\text { Interval }(\mathrm{cm})\end{array}$} & \multirow{2}{*}{ 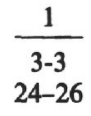 } & \multirow{2}{*}{$\frac{2}{3-3} \frac{}{64-66}$} & \multicolumn{2}{|c|}{3} & \multirow{2}{*}{$\frac{4}{3-4}$} & \multirow{2}{*}{$\frac{5}{4-1}$} & \multicolumn{2}{|c|}{6} & \multirow{2}{*}{$\frac{8}{5-1}$} & \multicolumn{2}{|c|}{10} & \multirow{2}{*}{ 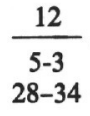 } & \multirow{2}{*}{ 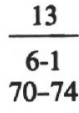 } \\
\hline & & & $\begin{array}{c}3-3 \\
89-91\end{array}$ & $\begin{array}{c}3-3 \\
116-120\end{array}$ & & & $\begin{array}{c}4-1 \\
111-115\end{array}$ & $\begin{array}{c}4-2 \\
36-40\end{array}$ & & $\begin{array}{c}5-2 \\
46-48\end{array}$ & $\begin{array}{c}5-2 \\
126-130\end{array}$ & & \\
\hline$q z$ & 0.00 & 0.00 & 0.00 & 0.00 & 0.00 & 0.00 & 0.27 & 0.00 & 0.00 & 0.00 & 0.00 & 0.00 & 0.00 \\
\hline or & 1.55 & 1.50 & 2.23 & 1.94 & 1.25 & 0.30 & 0.30 & 0.54 & 1.74 & 0.30 & 0.54 & 0.48 & 0.35 \\
\hline$a b$ & 20.25 & 21.15 & 20.38 & 21.05 & 22.10 & 20.39 & 20.51 & 20.53 & 20.83 & 20.75 & 20.59 & 20.70 & 19.30 \\
\hline an & 30.31 & 30.36 & 29.52 & 29.24 & 30.11 & 30.90 & 31.13 & 30.61 & 30.65 & 31.14 & 30.82 & 30.60 & 30.26 \\
\hline (wo & 12.46 & 12.22 & 12.78 & 12.35 & 11.62 & 11.99 & 11.86 & 12.53 & 13.04 & 12.04 & 12.52 & 12.55 & 13.22 \\
\hline di $\{$ en & 7.18 & 7.24 & 7.14 & 6.92 & 7.05 & 7.05 & 6.97 & 7.13 & 7.62 & 7.09 & 7.43 & 7.29 & 7.59 \\
\hline fs & 4.72 & 4.36 & 5.14 & 4.92 & 3.93 & 4.34 & 4.31 & 4.86 & 4.79 & 4.36 & 4.45 & 4.68 & 5.03 \\
\hline hv len & 10.55 & 10.11 & 8.99 & 9.21 & 10.94 & 11.82 & 12.34 & 10.93 & 9.21 & 11.91 & 10.50 & 10.30 & 11.94 \\
\hline$\left.{ }^{\text {hy }}\right|_{\text {fs }}$ & 6.93 & 6.10 & 6.47 & 6.55 & 6.09 & 7.28 & 7.64 & 7.46 & 5.79 & 7.31 & 6.29 & 6.61 & 7.91 \\
\hline / fo & 0.70 & 1.32 & 1.31 & 1.62 & 1.36 & 0.80 & 0.00 & 0.37 & 0.87 & 0.29 & 1.29 & 1.18 & 0.04 \\
\hline${ }^{0} \mathrm{fa}_{\mathrm{fa}}$ & 0.50 & 0.88 & 1.04 & 1.27 & 0.83 & 0.55 & 0.00 & 0.28 & 0.60 & 0.20 & 0.85 & 0.84 & 0.03 \\
\hline $\mathrm{mt}$ & 1.20 & 1.14 & 1.25 & 1.26 & 1.10 & 1.19 & 1.17 & 1.23 & 1.12 & 1.17 & 1.16 & 1.20 & 1.23 \\
\hline il & 2.82 & 2.86 & 2.81 & 2.80 & 2.86 & 2.70 & 2.82 & 2.81 & 2.88 & 2.83 & 2.85 & 2.85 & 2.57 \\
\hline ap & 0.33 & 0.34 & 0.34 & 0.34 & 0.34 & 0.31 & 0.34 & 0.34 & 0.36 & 0.34 & 0.34 & 0.34 & 0.26 \\
\hline Total & 99.51 & 99.57 & 99.50 & 99.47 & 99.59 & 99.63 & 99.67 & 99.60 & 99.50 & 99.73 & 99.63 & 99.62 & 99.73 \\
\hline $100 \mathrm{Mg} / \mathrm{Mg}+\mathrm{Fe}^{2+}$ & 61.44 & 63.17 & 59.48 & 59.83 & 64.82 & 63.13 & 62.72 & 60.58 & 62.07 & 62.91 & 63.41 & 61.97 & 61.80 \\
\hline D.I. ${ }^{a}$ & 21.81 & 22.65 & 22.61 & 22.99 & 23.36 & 20.69 & 21.08 & 21.07 & 22.57 & 21.05 & 21.13 & 21.18 & 19.66 \\
\hline pl(an) & 59.95 & 58.94 & 59.24 & 58.14 & 57.65 & 60.24 & 60.28 & 59.86 & 59.54 & 60.01 & 59.95 & 59.65 & 61.06 \\
\hline or & 2.98 & 2.83 & 4.27 & 3.71 & 2.36 & 0.58 & 0.58 & 1.04 & 3.27 & 0.58 & 1.05 & 0.93 & 0.72 \\
\hline$a b$ & 38.86 & 39.89 & 39.02 & 40.31 & 41.32 & 39.52 & 39.93 & 39.72 & 39.13 & 39.75 & 39.63 & 39.97 & 38.67 \\
\hline an & 58.16 & 57.28 & 56.71 & 55.98 & 56.30 & 59.89 & 59.93 & 59.23 & 57.59 & 59.67 & 59.32 & 59.10 & 60.62 \\
\hline
\end{tabular}

a Differentiation index (Thornton and Tuttle, 1960). 
Table 9. Major- and trace-element abundances of basalts from lower part of Hole 524.

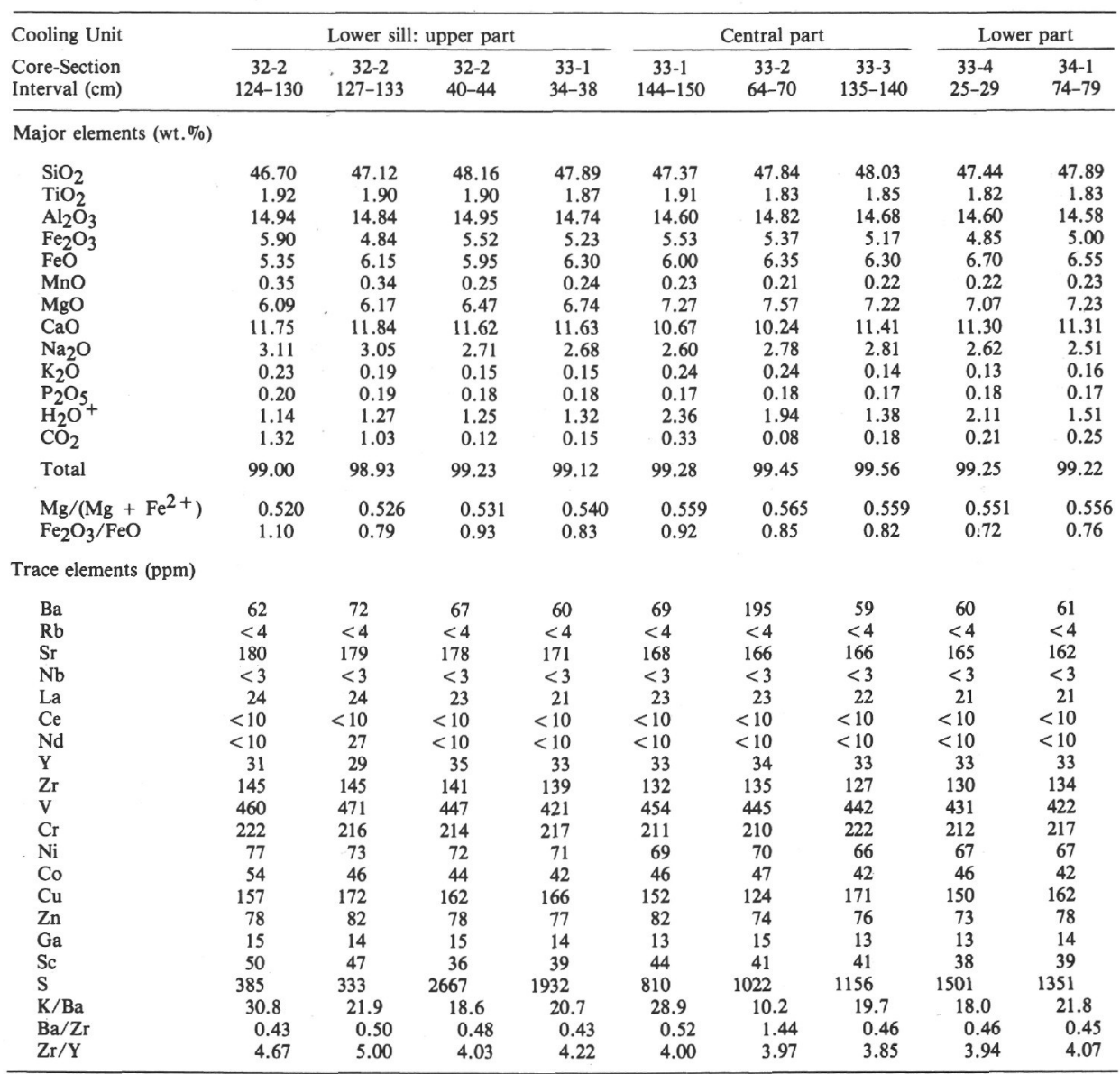

Table 10. Normative mineralogy (CIPW) of basalts from lower part of Hole 524 .

\begin{tabular}{|c|c|c|c|c|c|c|c|c|c|}
\hline \multirow{2}{*}{$\begin{array}{l}\text { Cooling Unit } \\
\text { Core-Section } \\
\text { Interval }(\mathrm{cm})\end{array}$} & \multicolumn{3}{|c|}{ Lower sill: upper part } & \multicolumn{4}{|c|}{ Central part } & \multicolumn{2}{|c|}{ Lower part } \\
\hline & $\begin{array}{c}32-2 \\
124-130\end{array}$ & $\begin{array}{c}32-2 \\
127-133\end{array}$ & $\begin{array}{c}32-3 \\
40-44\end{array}$ & $\begin{array}{c}33-1 \\
34-38\end{array}$ & $\begin{array}{c}33-1 \\
144-150\end{array}$ & $\begin{array}{c}33-2 \\
64-70\end{array}$ & $\begin{array}{c}33-3 \\
135-140\end{array}$ & $\begin{array}{c}33-4 \\
25-29\end{array}$ & $\begin{array}{c}34-1 \\
74-79\end{array}$ \\
\hline or & 1.43 & 1.18 & 0.91 & 0.91 & 1.47 & 1.46 & 0.85 & 0.79 & 0.97 \\
\hline$a b$ & 27.73 & 27.06 & 23.46 & 23.26 & 22.87 & 24.16 & 24.31 & 22.93 & 21.85 \\
\hline an & 27.53 & 27.52 & 28.84 & 28.46 & 28.54 & 27.99 & 27.63 & 28.64 & 28.86 \\
\hline wo & 9.91 & 10.83 & 11.76 & 11.92 & 9.67 & 9.38 & 11.66 & 11.17 & 10.90 \\
\hline $\operatorname{di}\{$ en & 4.96 & 5.49 & 5.99 & 6.15 & 5.16 & 5.02 & 6.20 & 5.85 & 5.76 \\
\hline$\left.\right|_{\mathrm{fs}}$ & 4.73 & 5.09 & 5.48 & 5.45 & 4.20 & 4.05 & 5.10 & 4.99 & 4.81 \\
\hline hy $\int_{\text {en }}$ & 2.70 & 3.01 & 5.16 & 4.74 & 7.44 & 5.95 & 3.93 & 5.60 & 7.45 \\
\hline${ }^{\text {hy }}$ \{fs $_{\text {f }}$ & 2.57 & 2.79 & 4.71 & 4.20 & 6.07 & 4.80 & 3.24 & 4.78 & 6.21 \\
\hline fo & 5.84 & 5.34 & 3.74 & 4.44 & 4.36 & 5.88 & 5.79 & 4.74 & 3.73 \\
\hline$\left.{ }^{\circ}\right\}_{f a}$ & 6.13 & 5.45 & 3.76 & 4.34 & 3.92 & 4.22 & 5.25 & 4.46 & 3.43 \\
\hline $\mathrm{mt}$ & 1.48 & 1.45 & 1.47 & 1.49 & 1.50 & 1.51 & 1.48 & 1.51 & 1.50 \\
\hline il & 3.84 & 3.78 & 3.69 & 3.64 & 3.77 & 3.57 & 3.59 & 3.57 & 3.58 \\
\hline ap & 0.50 & 0.47 & 0.44 & 0.44 & 0.42 & 0.44 & 0.41 & 0.44 & 0.41 \\
\hline Total & 99.35 & 99.47 & 99.41 & 99.44 & 99.40 & 99.42 & 99.44 & 99.47 & 99.46 \\
\hline $100 \mathrm{Mg} / \mathrm{Mg}+\mathrm{Fe}^{2+}$ & 51.95 & 52.65 & 53.13 & 53.98 & 55.94 & 56.54 & 55.85 & 55.08 & 55.64 \\
\hline D.I. ${ }^{a}$ & 29.16 & 28.24 & 24.37 & 24.17 & 24.34 & 25.62 & 25.16 & 23.72 & 22.83 \\
\hline $\mathrm{pl}(\mathrm{an})$ & 49.82 & 50.42 & 55.14 & 55.03 & 55.52 & 53.67 & 53.20 & 55.54 & 56.90 \\
\hline or & 2.53 & 2.11 & 1.70 & 1.73 & 2.79 & 2.72 & 1.60 & 1.52 & 1.88 \\
\hline$a b$ & 48.91 & 48.54 & 44.10 & 44.20 & 43.24 & 45.07 & 46.05 & 43.79 & 42.28 \\
\hline an & 48.56 & 49.35 & 54.20 & 54.08 & 53.97 & 52.21 & 52.35 & 54.70 & 55.83 \\
\hline
\end{tabular}

a Differentiation index (Thornton and Tuttle, 1960).

a rather primitive melt with a more evolved melt, probably trapped at the top of the magma chamber. The basalts of the other cooling units show a rather close geochemical relationship and cluster around the normal olivine ( \pm plagioclase) fractionation lines (Fig. 9B).
Cooling Unit 2 is chemically more primitive than other basalts from Hole 519A, as indicated by the $\mathrm{Mg}$ value of $0.67, \mathrm{Cr} \sim 390 \mathrm{ppm}, \mathrm{Ni} \sim 129 \mathrm{ppm}$, and low abundances of $\mathrm{TiO}_{2}$ (1.09 wt. \%), Y (18 ppm), $\mathrm{Zr}$ (65 ppm), V (250 ppm), Sr (100 ppm), Ga (9 ppm), and Sc 
Table 11. Major- and trace-element abundances of volcanic sedimentary layers in Hole 524 .

\begin{tabular}{|c|c|c|c|c|}
\hline $\begin{array}{l}\text { Core-Section } \\
\text { Interval }(\mathrm{cm})\end{array}$ & $\begin{array}{c}26-6 \\
61-63\end{array}$ & $\begin{array}{c}28-4 \\
108-110\end{array}$ & $\begin{array}{c}32-2 \\
35-38\end{array}$ & $\begin{array}{c}35-1 \\
102-104\end{array}$ \\
\hline \multicolumn{5}{|c|}{ Major elements (wt.\%) } \\
\hline $\mathrm{SiO}_{2}$ & 51.13 & 51.83 & 44.13 & 44.19 \\
\hline $\mathrm{TiO}_{2}$ & 0.95 & 1.89 & 1.17 & 2.09 \\
\hline $\mathrm{Al}_{2} \mathrm{O}_{3}$ & 13.91 & 15.40 & 13.42 & 13.98 \\
\hline $\mathrm{Fe}_{2} \mathrm{O}_{3}$ & 4.25 & 4.66 & 3.13 & 6.88 \\
\hline $\mathrm{FeO}$ & 0.75 & 2.80 & 2.35 & 1.80 \\
\hline $\mathrm{MnO}$ & 0.25 & 0.11 & 0.17 & 0.27 \\
\hline $\mathrm{MgO}$ & 1.70 & 3.19 & 2.95 & 4.92 \\
\hline $\mathrm{CaO}$ & 9.43 & 4.28 & 11.60 & 7.60 \\
\hline $\mathrm{Na}_{2} \mathrm{O}$ & 2.97 & 2.53 & 3.46 & 4.95 \\
\hline $\mathrm{K}_{2} \mathrm{O}$ & 3.52 & 5.41 & 2.67 & 0.92 \\
\hline $\mathrm{P}_{2} \mathrm{O}_{5}$ & 0.19 & 0.45 & 0.32 & 0.43 \\
\hline $\mathrm{H}_{2} \mathrm{O}^{+}$ & 2.79 & 4.29 & 6.64 & 4.76 \\
\hline $\mathrm{CO}_{2}$ & 7.47 & 2.09 & 7.07 & 6.33 \\
\hline Total & 99.31 & 98.93 & 99.08 & 99.12 \\
\hline
\end{tabular}

Trace elements (ppm)

\begin{tabular}{lrllr}
$\mathrm{Ba}$ & 240 & n.d. & n.d. & 265 \\
$\mathrm{Rb}$ & 52 & n.d. & n.d. & 11 \\
$\mathrm{Sr}$ & 191 & n.d. & n.d. & 140 \\
$\mathrm{Nb}$ & 78 & n.d. & n.d. & 28 \\
$\mathrm{La}$ & 26 & n.d. & n.d. & 28 \\
$\mathrm{Ce}$ & 72 & n.d. & n.d. & 34 \\
$\mathrm{Nd}$ & 24 & n.d. & n.d. & $\sim 12$ \\
$\mathrm{Y}$ & 26 & n.d. & n.d. & 8 \\
$\mathrm{Zr}$ & 525 & n.d. & n.d. & 234 \\
$\mathrm{~V}$ & 176 & n.d. & n.d. & 283 \\
$\mathrm{Cr}$ & 20 & n.d. & n.d. & 122 \\
$\mathrm{Ni}$ & 11 & n.d. & n.d. & 50 \\
$\mathrm{Co}$ & 13 & n.d. & n.d. & 192 \\
$\mathrm{Cu}$ & $<6$ & n.d. & n.d. & $<6$ \\
$\mathrm{Zn}$ & 127 & n.d. & n.d. & 68 \\
$\mathrm{Ga}$ & 19 & n.d. & n.d. & 6 \\
$\mathrm{Sc}$ & 9 & n.d. & n.d. & 28 \\
$\mathrm{~S}$ & 180 & n.d. & n.d. & 535 \\
\hline
\end{tabular}

Note: n.d. $=$ not determined.

(34 ppm). This is also consistent with the rare-earth-element pattern (Fig. 10), which has a relatively flat slope of the middle and heavy rare earths and the characteristic depletion of the light rare earths, with $(\mathrm{Ce} / \mathrm{Sm})_{\mathrm{N}}=$ 0.68 .

The effects of fractional crystallization of olivine and plagioclase are illustrated with several figures (Figs. 8A$\mathrm{C}$ and 9A and $\mathrm{B}$ ); variation diagrams are presented in which incompatible elements, such as $\mathrm{Ti}, \mathrm{Zr}, \mathrm{Y}$, and $\mathrm{V}$ are plotted against $\mathrm{Mg} / \mathrm{Mg}+\mathrm{Fe}^{2+}$.

Although the variation diagrams suggest that olivine fractionation was important in the evolution of the rocks, in the case of Hole 519A basalts, this seems to be inconsistent with a lack of olivine phenocrysts. According to Carman et al. (this vol.), one or more major mineral phases may occur as two types of phenocrysts in the basalts of Leg 73. One type is larger and more euhedral; the second is smaller and commonly intergrown with other major phases. The larger grains are considered to have crystallized slowly in a magma chamber and are designated herein as first generation. The smaller grains are often seriate down to groundmass size and are regarded as having formed as the magma rose to the sur-
Table 12. Major- and trace-element abundances of basalts from upper part of Hole 524

\begin{tabular}{|c|c|c|c|c|c|c|c|}
\hline \multirow{2}{*}{$\begin{array}{l}\text { Cooling Unit } \\
\text { Core-Section } \\
\text { Interval (cm) }\end{array}$} & \multicolumn{3}{|c|}{ Upper pillows } & \multicolumn{4}{|c|}{ Upper sill } \\
\hline & $\begin{array}{c}28-3 \\
64-69\end{array}$ & $\begin{array}{c}28-3 \\
93-97\end{array}$ & $\begin{array}{c}28-3 \\
105-109\end{array}$ & $\begin{array}{c}30-1 \\
123-128\end{array}$ & $\begin{array}{c}30-2 \\
72-76\end{array}$ & $\begin{array}{c}31-1 \\
80-84\end{array}$ & $\begin{array}{l}31-2 \\
3-7\end{array}$ \\
\hline \multicolumn{8}{|l|}{ Major elements (wt.\%) } \\
\hline $\mathrm{SiO}_{2}$ & 46.83 & 44.72 & 44.38 & 48.55 & 48.28 & 48.14 & 47.55 \\
\hline $\mathrm{TiO}_{2}$ & 4.31 & 4.24 & 4.21 & 3.52 & 3.50 & 3.56 & 3.62 \\
\hline $\mathrm{Al}_{2} \mathrm{O}_{3}$ & 14.95 & 14.63 & 14.57 & 14.35 & 14.17 & 14.21 & 14.18 \\
\hline $\mathrm{Fe}_{2} \mathrm{O}_{3}$ & 7.60 & 5.70 & 7.03 & 4.63 & 5.39 & 5.64 & 5.51 \\
\hline $\mathrm{FeO}$ & 4.65 & 7.50 & 5.60 & 7.05 & 6.70 & 6.40 & 6.70 \\
\hline $\mathrm{MnO}$ & 0.20 & 0.24 & 0.26 & 0.21 & 0.27 & 0.26 & 0.25 \\
\hline $\mathrm{MgO}$ & 2.92 & 4.02 & 3.96 & 4.75 & 5.14 & 4.99 & 5.03 \\
\hline $\mathrm{CaO}$ & 6.40 & 8.47 & 8.91 & 6.07 & 5.29 & 6.09 & 6.10 \\
\hline $\mathrm{Na}_{2} \mathrm{O}$ & 2.88 & 2.90 & 2.69 & 3.38 & 3.29 & 3.25 & 3.27 \\
\hline $\mathrm{K}_{2} \mathrm{O}$ & 3.71 & 2.29 & 2.32 & 2.93 & 3.92 & 2.91 & 2.67 \\
\hline $\mathrm{P}_{2} \mathrm{O}_{5}$ & 2.07 & 1.98 & 1.97 & 1.12 & 1.10 & 1.12 & 1.14 \\
\hline $\mathrm{H}_{2} \mathrm{O}^{+}$ & 1.88 & 1.71 & 2.20 & 2.43 & 1.91 & 1.84 & 2.53 \\
\hline $\mathrm{CO}_{2}$ & 0.29 & 0.22 & 0.31 & 0.09 & 0.19 & 0.22 & 0.14 \\
\hline Total & 98.69 & 98.62 & 98.41 & 99.08 & 99.15 & 98.63 & 98.69 \\
\hline $\begin{array}{l}\mathrm{Mg} /\left(\mathrm{Mg}+\mathrm{Fe}^{2+}\right) \\
\mathrm{Fe}_{2} \mathrm{O}_{3} / \mathrm{FeO}\end{array}$ & $\begin{array}{l}0.329 \\
1.63\end{array}$ & $\begin{array}{l}0.379 \\
0.76\end{array}$ & $\begin{array}{l}0.388 \\
1.26\end{array}$ & $\begin{array}{l}0.449 \\
0.66\end{array}$ & $\begin{array}{l}0.459 \\
0.80\end{array}$ & $\begin{array}{l}0.454 \\
0.88\end{array}$ & $\begin{array}{l}0.453 \\
0.82\end{array}$ \\
\hline \multicolumn{8}{|l|}{ Trace elements (ppm) } \\
\hline $\mathrm{Ba}$ & 1350 & 1334 & 1344 & 1034 & 1088 & 990 & 1027 \\
\hline $\mathrm{Rb}$ & 71 & 19 & 15 & 35 & 42 & 35 & 33 \\
\hline $\mathrm{Sr}$ & 659 & 672 & 661 & 498 & 415 & 461 & 489 \\
\hline $\mathrm{Nb}$ & 40 & 37 & 37 & 41 & 42 & 42 & 40 \\
\hline $\mathbf{L a}$ & 86 & 81 & 80 & 68 & 67 & 68 & 69 \\
\hline $\mathrm{Ce}$ & 141 & 133 & 155 & 118 & 100 & 114 & 106 \\
\hline $\mathrm{Nd}$ & 67 & 67 & 73 & 54 & 52 & 42 & 54 \\
\hline $\mathrm{Y}$ & 41 & 42 & 41 & 36 & 35 & 37 & 36 \\
\hline$z_{5}$ & 261 & 255 & 248 & 285 & 287 & 283 & 275 \\
\hline $\mathrm{v}$ & 348 & 323 & 309 & 231 & 195 & 227 & 198 \\
\hline $\mathrm{Cr}$ & 21 & 18 & 21 & $<10$ & $<10$ & 13 & $<10$ \\
\hline $\mathrm{Ni}$ & 14 & 14 & 13 & $<3$ & $<3$ & $<3$ & $<3$ \\
\hline Co & 29 & 43 & 49 & 29 & 23 & 26 & 28 \\
\hline $\mathrm{Cu}$ & 7 & 9 & 11 & $<4$ & $<4$ & $<4$ & $<4$ \\
\hline $\mathrm{Zn}$ & 135 & 123 & 131 & 120 & 107 & 118 & 118 \\
\hline $\mathrm{Ga}$ & 18 & 18 & 17 & 19 & 19 & 20 & 19 \\
\hline $\mathrm{Sc}$ & 37 & 33 & 33 & 25 & 25 & 25 & 25 \\
\hline $\mathrm{s}$ & 329 & 3601 & 3606 & 44 & 1477 & 465 & 89 \\
\hline $\mathrm{K} / \mathrm{Ba}$ & 22.8 & 14.3 & 14.3 & 23.5 & 30.0 & 24.4 & 21.6 \\
\hline $\mathrm{K} / \mathbf{R b}$ & 433 & 1000 & 1280 & 690 & 770 & 690 & 670 \\
\hline $\mathrm{Ba} / \mathrm{Nb}$ & 33.8 & 36.1 & 36.3 & 25.2 & 25.9 & 23.6 & 25.7 \\
\hline $\mathrm{Ba} / \mathrm{Zr}$ & 5.17 & 5.23 & 5.42 & 3.63 & 3.79 & 3.50 & 3.73 \\
\hline $\mathrm{Zr} / \mathrm{Nb}$ & 6.5 & 6.9 & 6.7 & 6.9 & 6.8 & 6.7 & 6.9 \\
\hline
\end{tabular}

Table 13. Normative mineralogy (CIPW) of basalts from upper part of Hole 524.

\begin{tabular}{|c|c|c|c|c|c|c|c|}
\hline \multirow{2}{*}{$\begin{array}{l}\text { Cooling Unit } \\
\text { Core-Section } \\
\text { Interval (cm) }\end{array}$} & \multirow{2}{*}{$\frac{1}{28-3}$} & \multirow{2}{*}{$\frac{2}{28-3}$} & \multirow{2}{*}{ 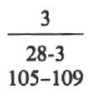 } & \multicolumn{4}{|c|}{ Upper sill } \\
\hline & & & & $\begin{array}{c}30-1 \\
123-128\end{array}$ & $\begin{array}{c}30-2 \\
72-76\end{array}$ & $\begin{array}{c}31-1 \\
80-84\end{array}$ & $\begin{array}{l}31-2 \\
3-7\end{array}$ \\
\hline c & 0.20 & 0.00 & 0.00 & 0.00 & 0.00 & 0.00 & 0.00 \\
\hline or & 22.80 & 14.04 & 14.35 & 17.95 & 23.93 & 17.86 & 16.46 \\
\hline$a b$ & 25.35 & 25.45 & 23.83 & 29.65 & 28.76 & 28.56 & 28.87 \\
\hline an & 17.05 & 20.89 & 21.81 & 15.90 & 12.73 & 16.19 & 16.83 \\
\hline (wo & 0.00 & 3.27 & 3.73 & 2.99 & 2.39 & 2.56 & 2.53 \\
\hline di en & 0.00 & 1.39 & 1.64 & 1.43 & 1.16 & 1.24 & 1.22 \\
\hline$l_{\mathrm{fs}}$ & 0.00 & 1.89 & 2.08 & 1.51 & 1.19 & 1.28 & 1.27 \\
\hline (en & 6.28 & 3.17 & 4.07 & 4.71 & 0.41 & 5.43 & 5.34 \\
\hline hy $\left.\right|_{\mathrm{fs}}$ & 9.98 & 4.30 & 5.17 & 4.96 & 0.42 & 5.63 & 5.56 \\
\hline fo & 0.90 & 4.08 & 3.24 & 4.29 & 8.17 & 4.38 & 4.57 \\
\hline${ }^{o l}\{f a$ & 1.58 & 6.10 & 4.53 & 4.98 & 9.24 & 5.00 & 5.25 \\
\hline $\mathrm{mt}$ & 1.57 & 1.73 & 1.65 & 1.53 & 1.57 & 1.57 & 1.60 \\
\hline il & 8.51 & 8.35 & 8.37 & 6.93 & 6.87 & 7.02 & 7.17 \\
\hline ap & 5.10 & 4.86 & 4.89 & 2.75 & 2.69 & 2.76 & 2.82 \\
\hline Total & 99.32 & 99.52 & 99.36 & 99.58 & 99.51 & 99.48 & 99.49 \\
\hline $100 \mathrm{Mg} / \mathrm{Mg}+\mathrm{Fe}^{2+}$ & 32.85 & 37.94 & 38.87 & 44.87 & 45.97 & 45.42 & 45.25 \\
\hline D.I. ${ }^{a}$ & 48.15 & 39.49 & 38.18 & 47.61 & 52.69 & 46.42 & 45.33 \\
\hline $\mathrm{pl}(\mathrm{an})$ & 40.22 & 45.08 & 47.78 & 34.90 & 30.68 & 36.18 & 36.82 \\
\hline or & 34.97 & 23.2 & 23.9 & 28. & 36.58 & 28.52 & 26.48 \\
\hline$a b$ & 38.88 & 42.16 & 39.72 & 46.70 & 43.96 & 45.61 & 46.44 \\
\hline an & 26.15 & 34.60 & 36.35 & 25.03 & 19.45 & 25.86 & 27.07 \\
\hline
\end{tabular}

a Differentiation index (Thornton and Tuttle, 1960).

face (second generation). In the case of olivine there actually appears to have been a hiatus in its crystallization in the rocks of other holes of Leg 73, as discussed in Carman et al. (this vol.), and if olivine formed early in the rocks of Hole 519A it would appear to have formed a first generation of crystals that were separated from 


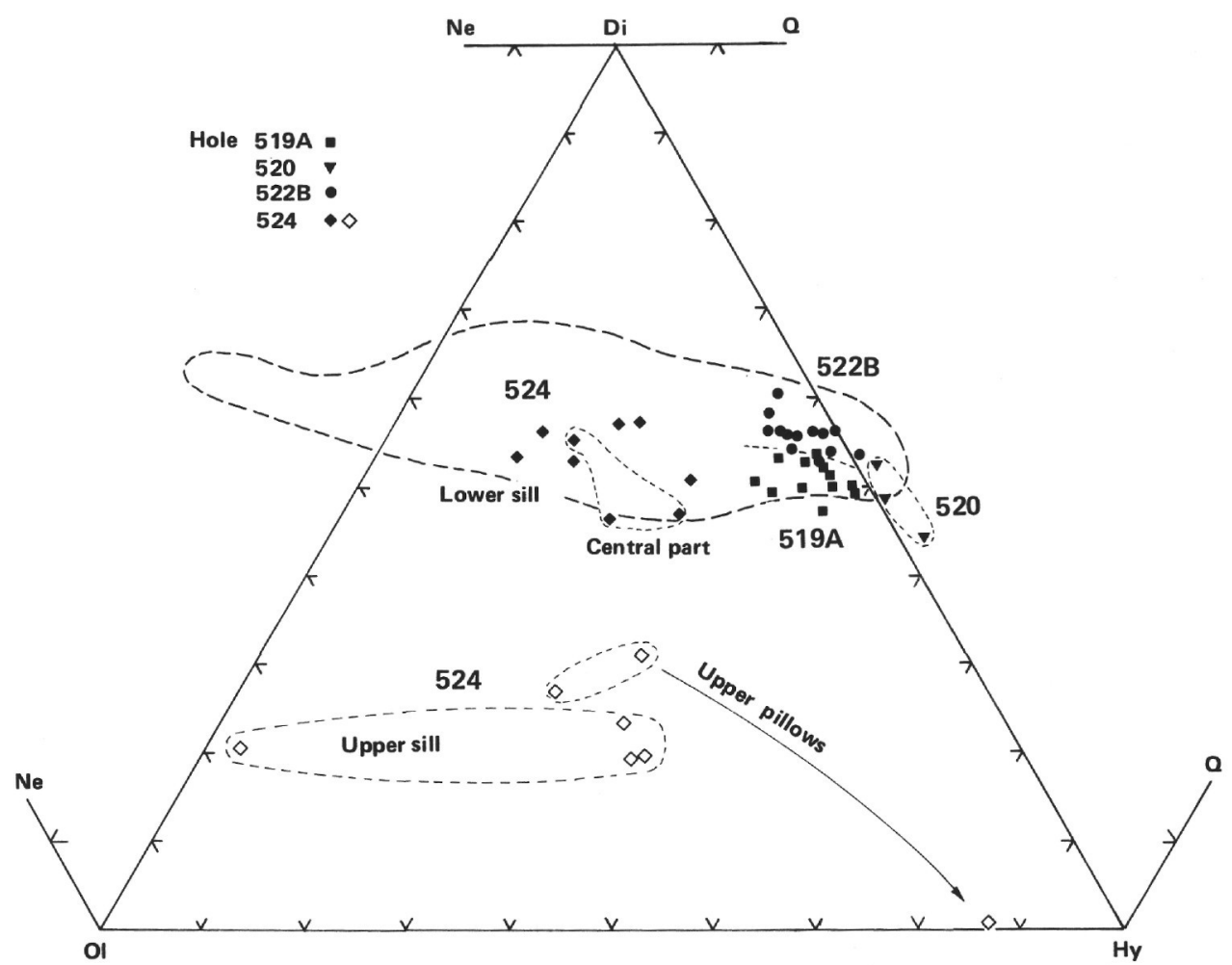

Figure 5. CIPW normative compositions of Hole 519A, 520, 522B, and 524 basalts, plotted in the system diopside-olivine-hypersthene ( + quartz, nepheline). Field of normal mid-ocean-ridge basalts (bold dashed line) is from Thompson et al. (1972).

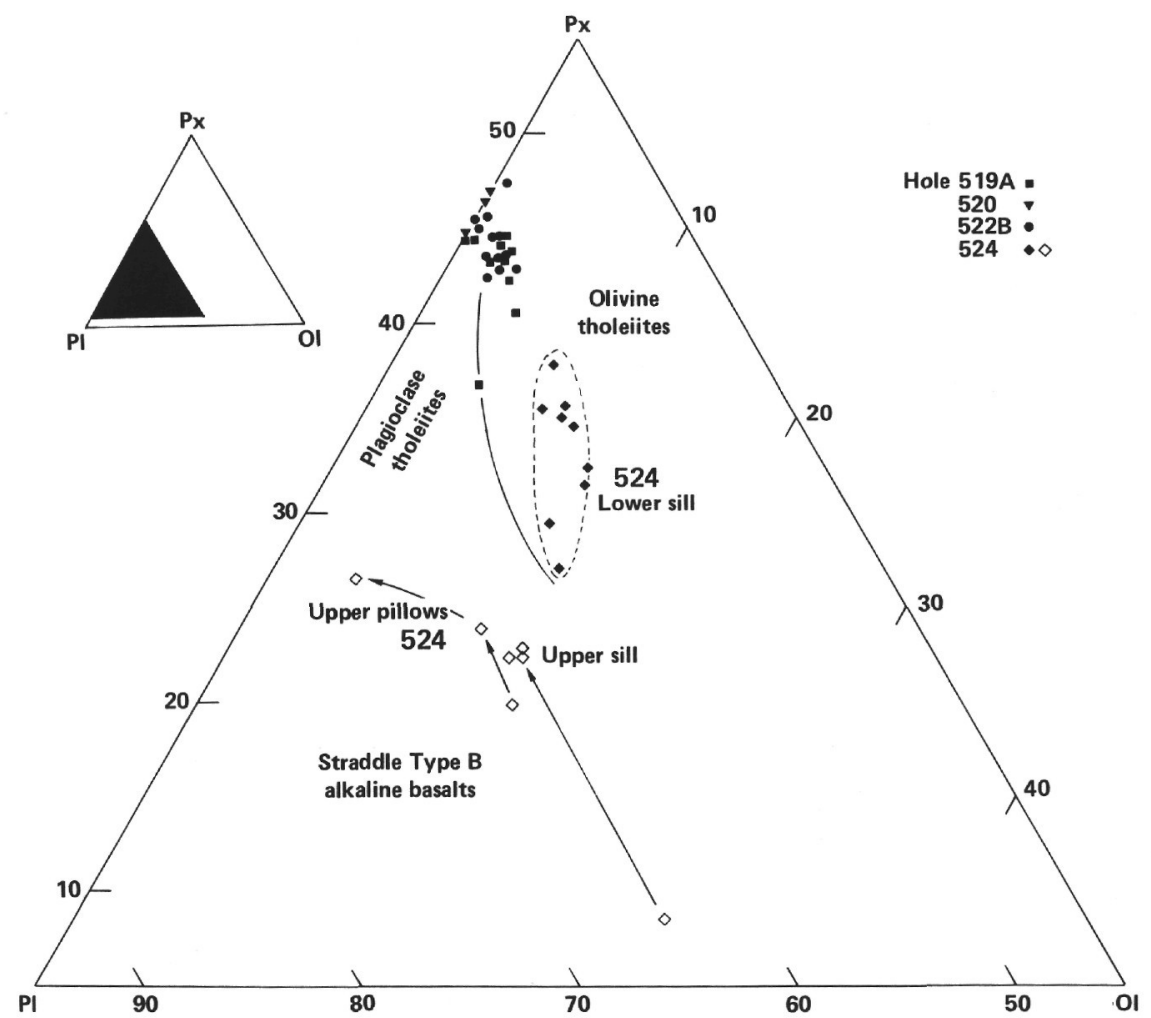

Figure 6. Normative olivine-plagioclase-pyroxene relationships in the Leg 73 basalts. The inferred olivine-plagioclase cotectic line is from Shido et al. (1971); the straddle Type B classification is after Miyashiro (1978). 


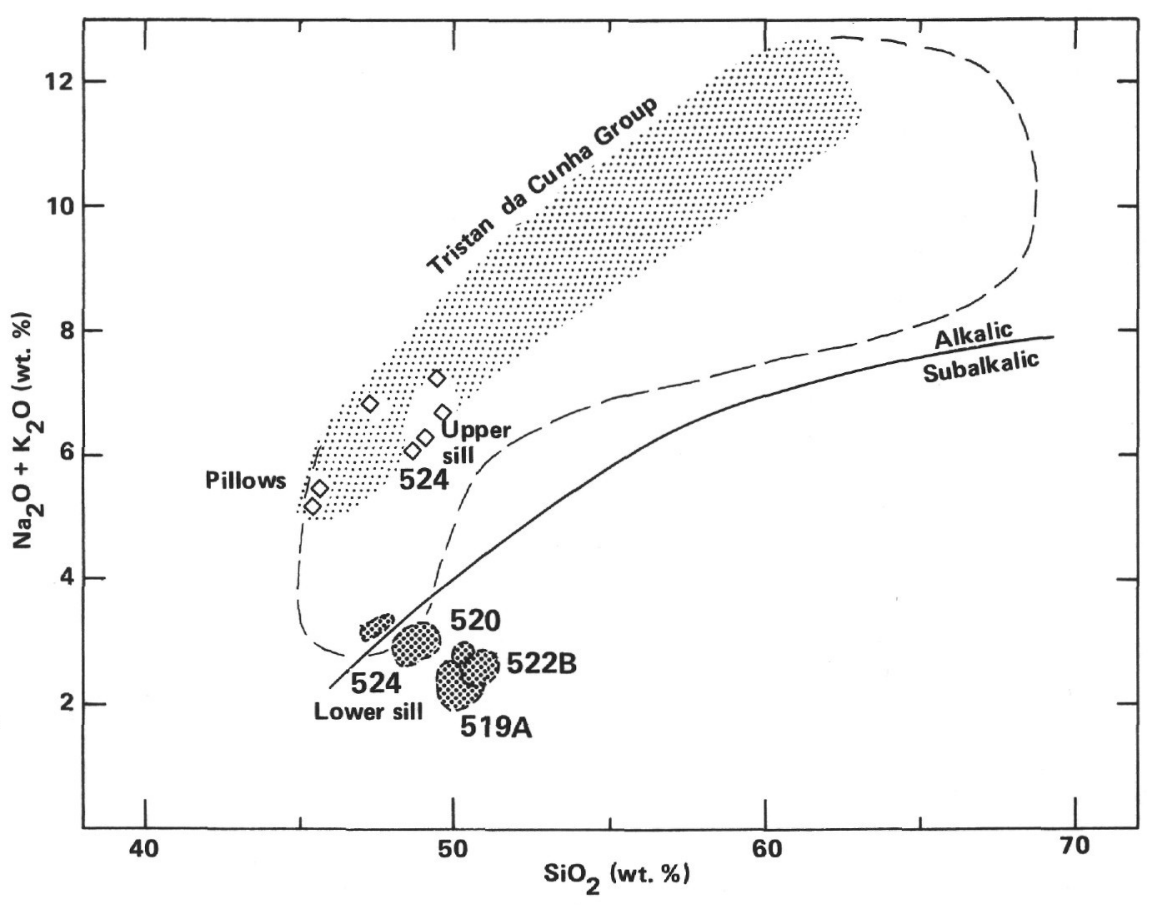

Figure 7. Alkali-silica variation diagram of Leg 73 basalts. Boundary between alkalic and subalkalic rocks after McDonald and Katsura (1964). Dashed line indicates field of straddle type alkaline associations (Miyashiro, 1978). Field of alkaline volcanics of Tristan da Cunha group volcanoes is from Smith (1930), Dunne (1941), and Baker et al. (1964).

the magma that finally reached the surface. Only plagioclase and spinel have been detected as first generation phenocrysts. Plagioclase and olivine appear as second generation phenocrysts; quench phases occur as rare olivine plus abundant plagioclase, clinopyroxene, and titanomagnetite.

Geochemistry and the crystallization sequence (Carman et al., this vol.) seem to indicate that Cooling Units $2,2 a, 3$, and 4 are sequentially more evolved, in that order. However, this is in the reverse order of eruption (Fig. 2), unless the lower units are intrusive. Glassy and brecciated tops and fine quench textures refute the notion of intrusion for any of these units. The alternative explanation is a gradual draining of a zoned magma chamber from an upper more evolved part (Cooling Unit 4) to a lower more primitive part (Cooling Unit 2). In addition, mixing effects between evolved and very primitive magmas within the magma chamber cannot be excluded.

Cooling Unit 1 probably terminated the eruption cycle. It contains the freshest basalts in Hole $519 \mathrm{~A}\left(\mathrm{Fe}_{2} \mathrm{O}_{3} /\right.$ $\mathrm{FeO}=0.15-0.43)$. The high contents of $\mathrm{TiO}_{2}(1.28$ wt. \%), Y (22 ppm), Zr (84 ppm), V (299 ppm), Co (39 $\mathrm{ppm}), \mathrm{Sc}(44 \mathrm{ppm})$, and REEs, together with a slight enrichment of the LREEs $\left((\mathrm{Ce} / \mathrm{Sm})_{\mathrm{N}}=0.70\right.$; Fig. 10), demonstrate that these basalts are relatively evolved. Quench textures and glassy margins indicate that the upper $70 \mathrm{~cm}$ of Cooling Unit 1 are made up of pillow lava. Thus, Cooling Unit 1 may represent the evolution of the Cooling Unit 2 magma beyond the stages reached by Cooling Units 2 a through 4.

The discussion of the magmatic origin of the Hole 519A basalts can be based on some ratios: the $\mathrm{Ti} / \mathrm{V}$ ra- tio (Fig. 11; as demonstrated by Shervais, 1982) and the ratios of the more-to-less-HYG elements (Saunders et al., 1980; and Tarney et al., 1979), such as Y/Zr (Fig. 12), $\mathrm{Ba} / \mathrm{Zr}$ (Fig. 13), and $\mathrm{Ce} / \mathrm{Y}$. These ratios seem to be unaffected by the low-pressure fractional crystallization of olivine, clinopyroxene, and plagioclase and thus may reflect the ratios in primary MORB melts. Alternatively, different degrees of dynamic or continuous melting of mantle material could produce primary magmas that differ in $\mathrm{Zr} / \mathrm{Y}$ and $\mathrm{Ce} / \mathrm{Yb}$ ratios from each other as well as from their initial source (Langmuir et al., 1977).

The ratios of $\mathrm{Ba} / \mathrm{Zr}$ (Table 2), $\mathrm{Ba} / \mathrm{La}$ (Table 3), and the $\mathrm{Sr} / \mathrm{Ca}-\mathrm{Ba} / \mathrm{Ca}$ systematics (Fig. 14), as well as the similarity of the REE patterns of all the basalts in Hole 519A (Fig. 10) expressed by the LREE slopes $(\mathrm{Ce} / \mathrm{Sm})_{\mathrm{N}}$ and the $\mathrm{Ce} / \mathrm{Yb}$ ratios (Table 3) indicate that Cooling Units 1 to 4 may have been derived from a very similar magma batch, which differs slightly from that of the older Cooling Unit 5.

The composition of a primary magma from which the Hole 519A N-type MORBs originated may have been similar to the composition of other Mid-Atlantic Ridge segments, as discussed in Dietrich et al. (1981). The trace-element ratios of the Hole 519A basalts (Figs. 12-14) seem to suggest a large degree of partial melting of lherzolitic mantle material that is depleted in the more-HYG elements relative to chondrites. In addition, close relationships exist with the trace-element ratios of the Paleogene Gorgona komatiites (Figs. 12-14), which have been regarded as a primary magma for MORB (Dietrich et al., 1981). From the $\mathrm{Yb}$ content of the komatiites $(\sim 1.5 \mathrm{ppm})$, it might be inferred that approximately $18 \%$ of partial melting of a depleted lherzo- 

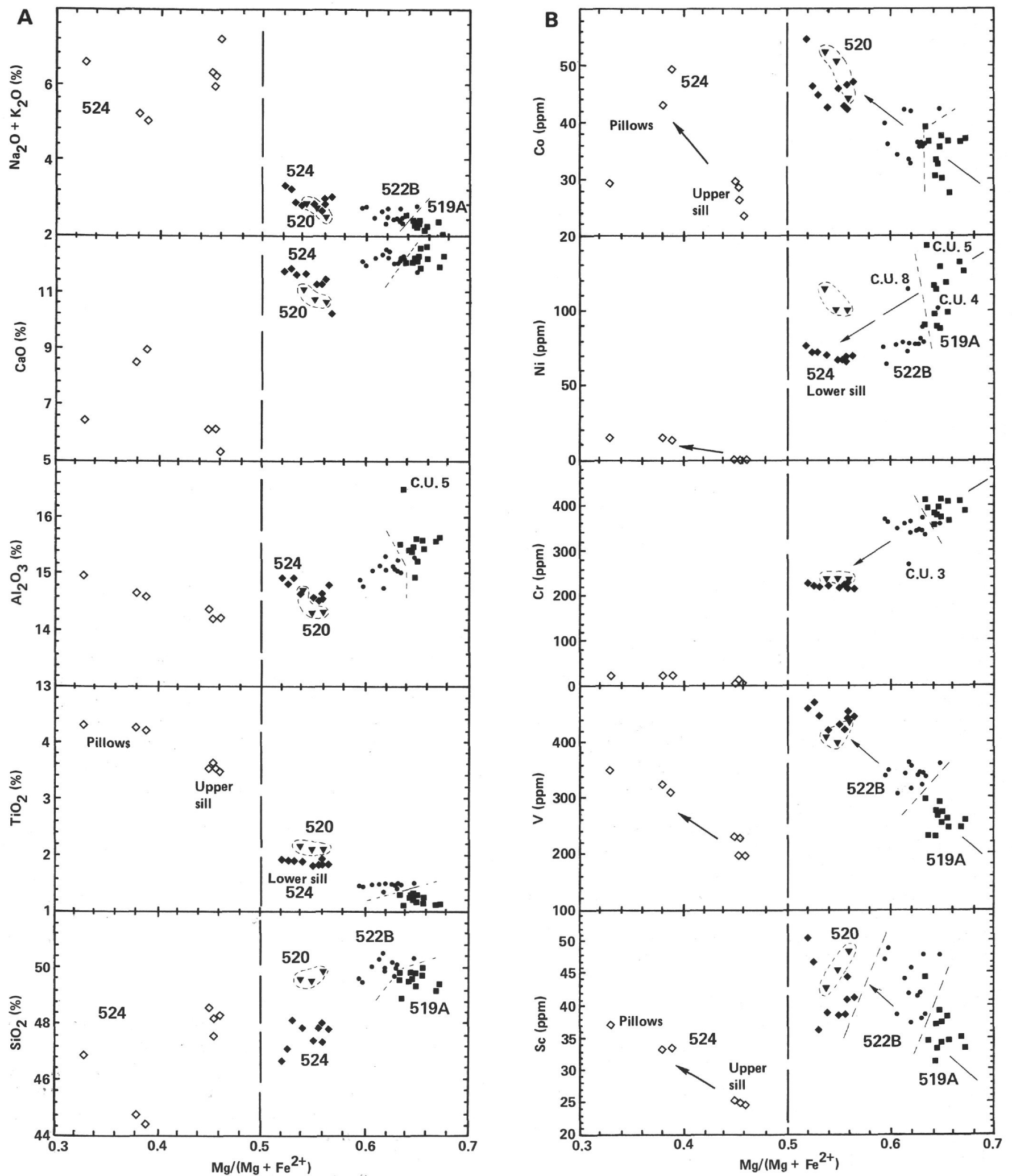

Figure 8. Compositional relationships among Leg 73 basalts from Holes $519 \mathrm{~A}, 520,522 \mathrm{~B}$, and $524 . \mathrm{Mg} /\left(\mathrm{Mg}+\mathrm{Fe}^{2+}\right)$ values calculated on a calciteand water-free basis on the assumption that $\mathrm{Fe}_{2} \mathrm{O}_{3} / \mathrm{FeO}=0.1$. C.U. = Cooling Unit. A. Major elements. B. Transitional trace elements. Arrows $=$ fractionation control lines. C. Hygromagmatophile trace elements. Arrows $=$ fractionation control lines. 


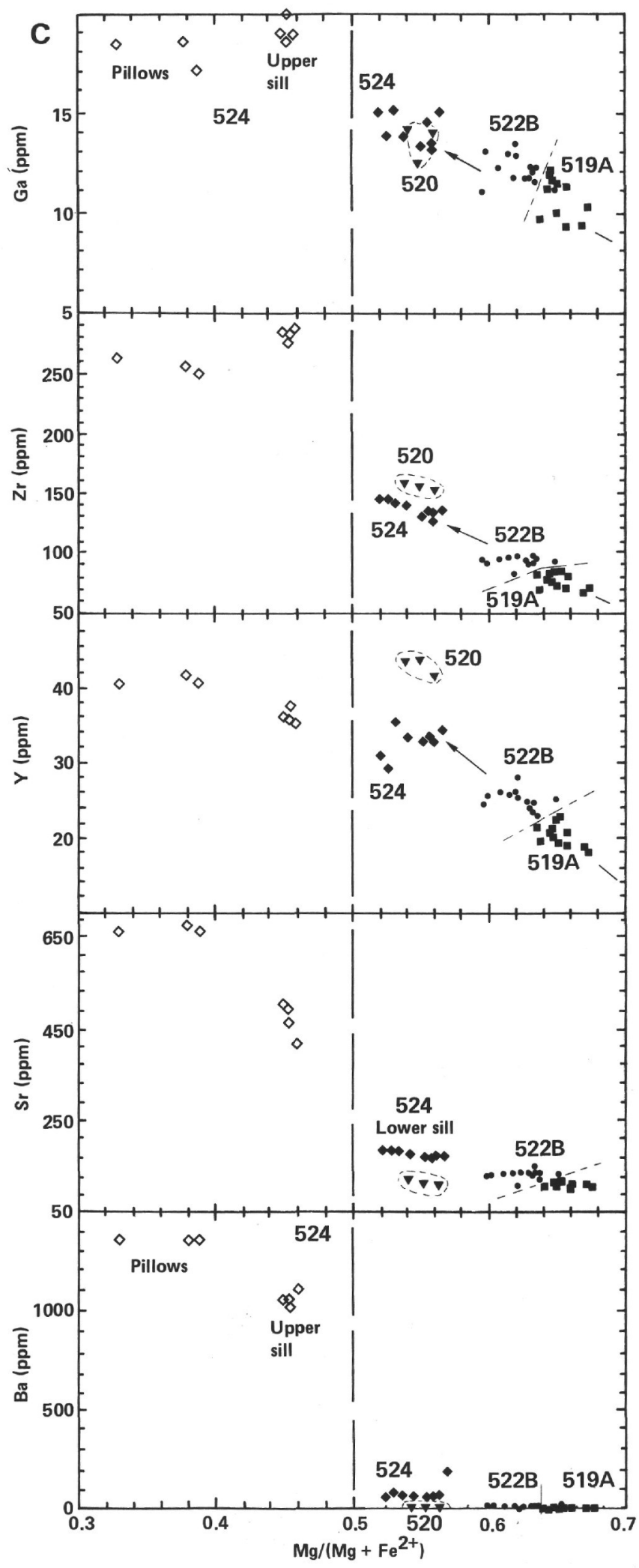

Figure 8. (Continued). litic source is necessary to produce such a primary MORB liquid (O’Nions et al., 1978).

\section{Conclusions}

The major- and trace-element characteristics of the early Pliocene basalts from Hole 519A at the eastern flank of the Mid-Atlantic Ridge are very similar to typical N-type MORB. According to evidence from the spectra of incompatible, or less-hygromagmatophile elements, such as $\mathrm{Ti}, \mathrm{V}, \mathrm{Y}$, and $\mathrm{Zr}$ and to evidence from REE abundances, these basalts are the result of a lowpressure fractionation of olivine, spinel, and plagioclase prior to eruption. Cooling Unit 2 exhibits the most primitive tholeiitic characteristics $\left(\mathrm{Mg}\right.$ value $=0.67, \mathrm{TiO}_{2}$ $=1.09$ wt. $\%, \mathrm{Cr}=390 \mathrm{ppm}$, and $\mathrm{Ni}=129 \mathrm{ppm}$ ) and thus overlaps in composition the primitive olivine tholeiites from other normal Mid-Atlantic Ridge segments.

\section{Hole 520}

Hole 520 is on the eastern flank of the present MidAtlantic Ridge crest close to Hole 519A (Fig. 1). The basaltic basement reached in Hole 520 is probably late Miocene in age or slightly older and thus is similar in age to the basalts encountered in Hole 519A.

In total, $10.5 \mathrm{~m}$ of basalt were drilled, and $1.7 \mathrm{~m}$ of basalt were recovered. The samples from this hole all consist of separate pieces. None could be shown to be contiguous, and thus no stratigraphy could be established. However, the presence of glass in six of eight pieces recovered, together with the fine quench texture of all the pieces and the poor recovery, suggests a pillowed sequence.

Only three basalt samples were chosen for complete analysis (Tables 3,5 , and 6). They all show very similar chemical characteristics: $\mathrm{TiO}_{2}=2.11$ to 2.18 wt. \%, $\mathrm{K}_{2} \mathrm{O}=0.1$ to 0.39 wt. $\%, \mathrm{P}_{2} \mathrm{O}_{5}=0.20$ to 0.21 wt. $\%, \mathrm{Zr}$ $=153$ to $158 \mathrm{ppm}, \mathrm{Y}=42$ to $44 \mathrm{ppm}, \mathrm{V}=400$ to 438 $\mathrm{ppm}, \mathrm{Co}=44$ to $52 \mathrm{ppm}, \mathrm{Sc}=43$ to $48 \mathrm{ppm}$, and $\mathrm{Ba}=$ 7 to $11 \mathrm{ppm}$. Despite low amounts of $\mathrm{CO}_{2}$, the relatively high ferric/ferrous ratios of the basalts (0.44-1.83) suggest that the samples are altered. They are quartz normative (Table 6 and Fig. 5) and can be classified as plagioclase tholeiites (Fig. 6) according to their normative compositions.

From the different chemical variation diagrams $(\mathrm{Mg}$ values versus $\mathrm{TiO}_{2}, \mathrm{P}_{2} \mathrm{O}_{5}, \mathrm{Zr}, \mathrm{Y}, \mathrm{V}, \mathrm{Co}$, and Sc;Figs. $8 \mathrm{~A}-\mathrm{C})$ and from the high REE contents, it is apparent that the Hole 520 basalts have crystallized from fairly evolved (N-type MORB) tholeiitic melts.

The relatively low amounts of $\mathrm{CaO}$ (10.6-11.09 wt. $\%$ ), $\mathrm{Al}_{2} \mathrm{O}_{3}(14.3-14.7$ wt. \%), and $\mathrm{Sr}(104-118 \mathrm{ppm})$ relative to the low $\mathrm{Mg}$ values of 0.54 to 0.56 , as well as the negative Eu anomaly (Fig. 10), suggest a plagioclase fractionation prior to eruption of the melts. The same effects can also be demonstrated in Figure 9A.

According to the $\mathrm{Zr} / \mathrm{Y}$ ratios of 3.55 to 3.64 (Fig. 12) and $\mathrm{Ba} / \mathrm{Zr}$ ratios of 0.044 to 0.072 (Fig. 13), the magmas of the ridge segment at Hole 520 originated from slightly different depleted mantle source material than the Hole 519 basalts. This is particularly evident in the high Ni contents of the Hole 520 basalts (Fig. 8B). 

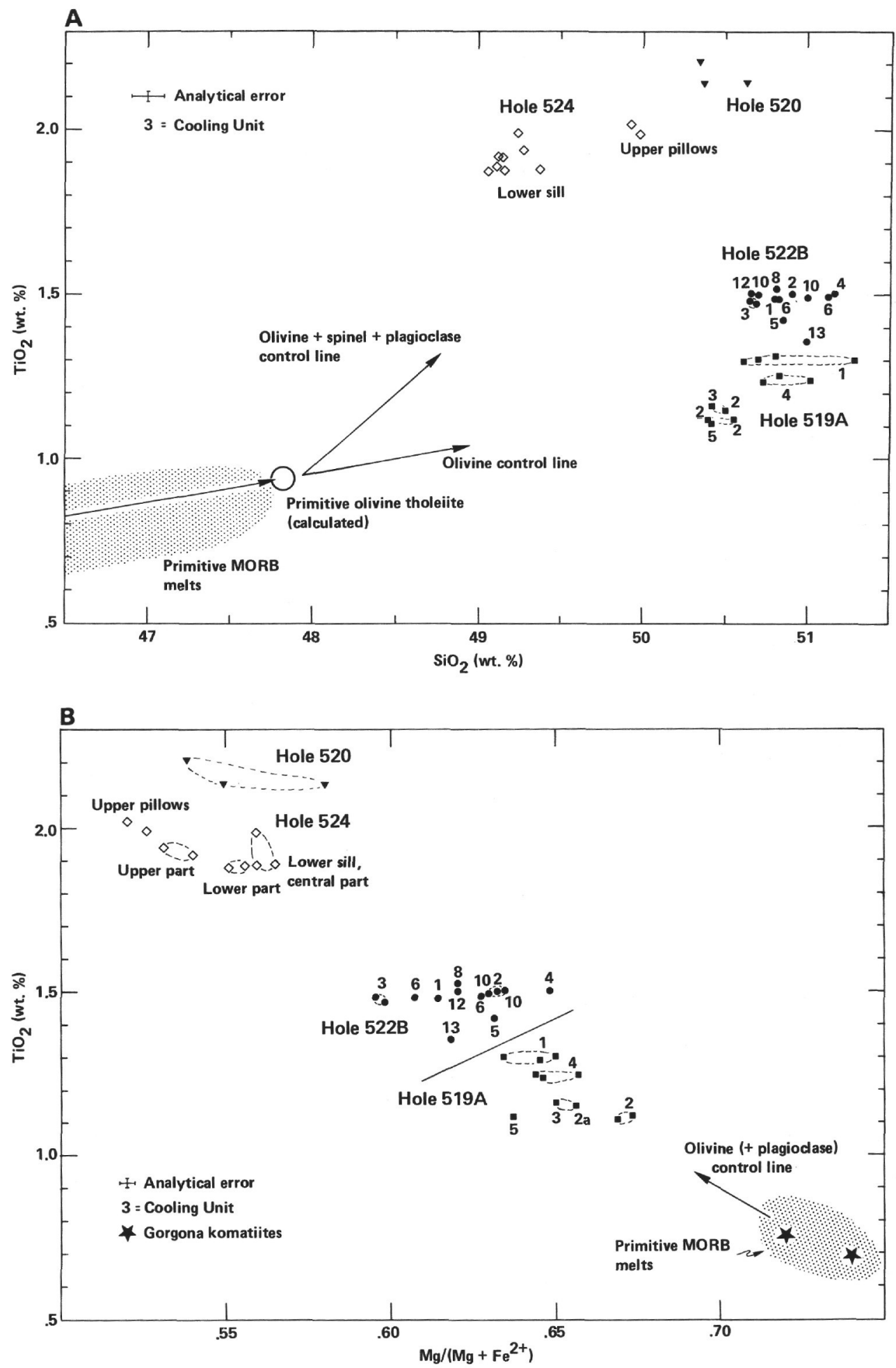

Figure 9. A. Enlarged $\mathrm{SiO}_{2}$ versus $\mathrm{TiO}_{2}$ variation diagram of Hole 519A, 520, 522B, and 524 tholeitic basalts. $\mathrm{TiO}_{2}$ and $\mathrm{SiO}_{2}$ recalculated on a calcite- and water-free basis. Primitive olivine-tholeiite composition derived from Gorgona pyroxenitic komatiites by fractionation (left arrow) of 20 to $25 \%$ olivine and $\approx 0.5 \%$ spinel (Dietrich et al., 1981). Composition of primitive MORB melts is also from Dietrich et al. (1981). See text for discussion. B. Enlarged $\mathrm{TiO}_{2}$ versus $\mathrm{Mg} /\left(\mathrm{Mg}+\mathrm{Fe}^{2+}\right)$ variation diagram of Hole 519A, 520, 522B, and 524 tholeiitic basalts. $\mathrm{TiO}_{2}$ and $\mathrm{Mg}$ number recalculated on a calcite- and water-free basis. Compositions of primitive MORB melts and of Gorgona komatiites in Dietrich et al. (1981). 


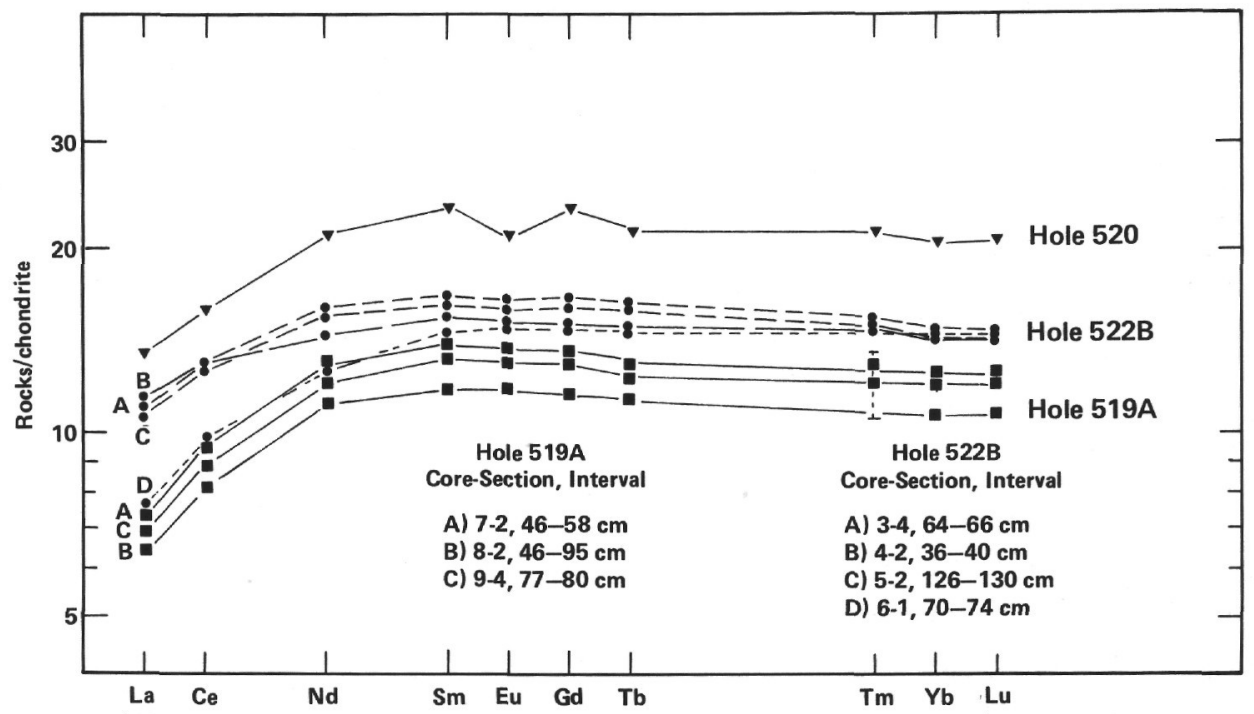

Figure 10. Chondrite-normalized REE plot for the basalts and diabases from Holes 519A, 520, and 522B. Normalizing values: Leedy chondrite/1.2 (Masuda et al., 1973); Tb and Tm values from Haskin et al. (1968).

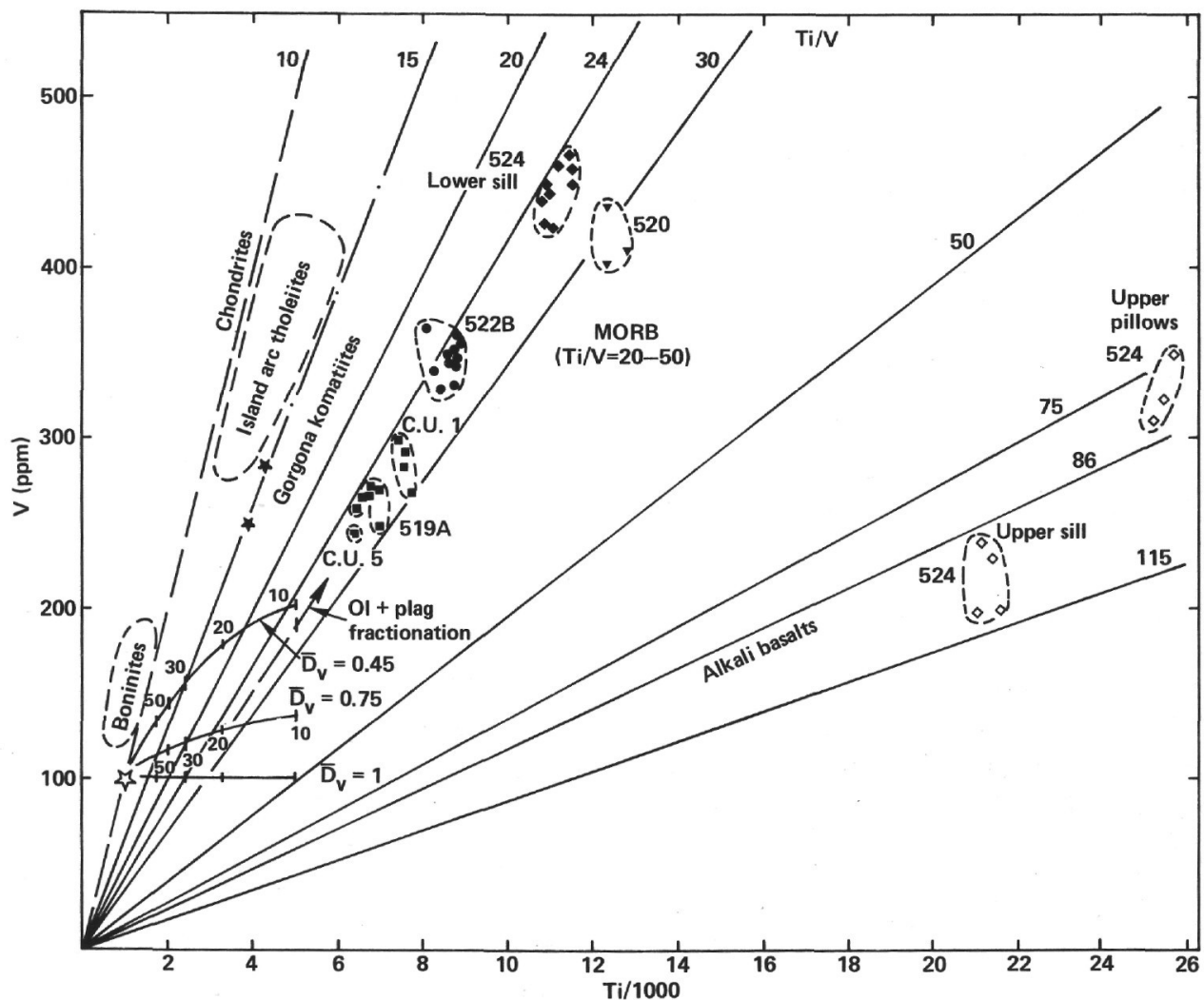

Figure 11. Ti-V relationships for Leg 73 basalts compared with chrondrites (Mason, 1971), bulk earth (open star; Pearce and Cann, 1973), komatiites (solid stars; Dietrich et al., 1981), boninites (Dietrich et al., 1978; and Dietrich, unpublished data), island arc tholeiites (Shervais, 1982), and MORB (Langmuir et al., 1977; and Shervais, 1982). Straight arrow indicates path of fractional crystallization; curves show degrees of partial melting of lherzolite with different bulk partition coefficients for V, Dv (after Shervais, 1982). C.U. = Cooling Unit. 


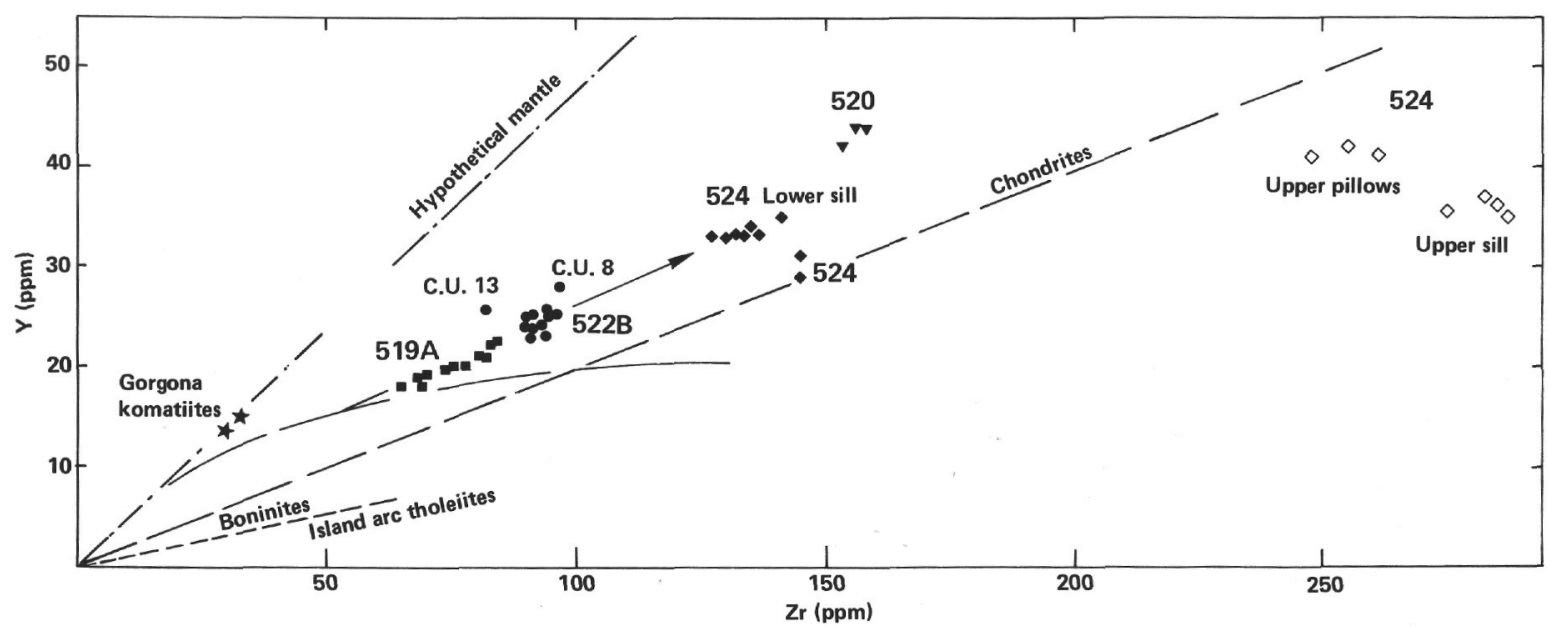

Figure 12. Y versus $\mathrm{Zr}$ plot for Leg 73 basalts. The figure illustrates the possible genetic relationships between the different basaltic rocks from Holes 519A, 520, 522B, and 524; the results of partial melting are shown by the curve, and the results of fractional crystallization are shown by the straight arrow. Data for hypothetical mantle from Jagoutz et al. (1979); for Gorgona komatiites from Dietrich et al. (1981); for chondrites from Mason (1971); for boninites from Dietrich et al. (1978); and for island arc basalts from Pearce and Norry (1979) and Gill (1981). C.U. = Cooling Unit.

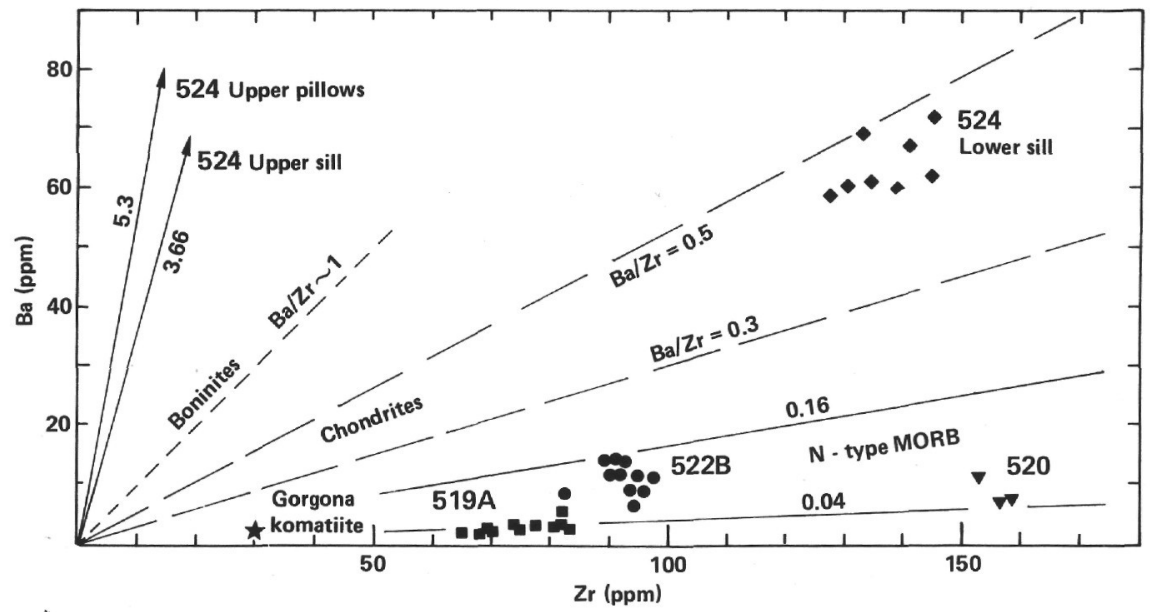

Figure 13. Ba versus $\mathrm{Zr}$ plot for Leg 73 basalts. Sources of data for boninites, chondrites, and komatiites as in Fig. 12.

\section{Hole 522B}

\section{Discussion}

Hole 522B was drilled approximately $1000 \mathrm{~km}$ east of the present crest of the Mid-Atlantic Ridge (Fig. 1) at magnetic Anomaly 16, which has a corresponding age of 38 m.y. (late Eocene).

In total, $19 \mathrm{~m}$ of basaltic pillow lavas and flows were penetrated in Hole 522B (Fig. 3). Thirteen cooling units were distinguished on the basis of the glassy margins and fine quench textures.

The variation of major-element compositions for the 13 samples selected for analysis is presented in Table 7 and Figure 8A. Most of the basaltic rocks are slightly olivine normative (Table 8 ) and very similar to the tholeiitic basalts of Hole 519A (Figs. 5 and 6), although the latter are lower in the normative diopsidic components.
The lowest and thus oldest cooling unit, Cooling Unit 13 , is quite different in composition from the other pillow lavas and flows (Figs. 8 and 9). Despite its partly evolved state $\left(\mathrm{SiO}_{2}=50 \mathrm{wt} . \%, \mathrm{Mg}\right.$ value $=0.62, \mathrm{Cr}=$ $262 \mathrm{ppm}$, and $\mathrm{Ni}=73 \mathrm{ppm}$ ), this cooling unit has the most primitive $\mathrm{N}$-type MORB characteristics of the Hole 522B basalts, with $\mathrm{K}_{2} \mathrm{O}=0.06$ wt. $\%, \mathrm{TiO}_{2}=1.34$ wt. $\%, \mathrm{Zr}=82 \mathrm{ppm}, \mathrm{Y}=26 \mathrm{ppm}$, and $\mathrm{Ba}=7 \mathrm{ppm}$. The fractionation of plagioclase plus olivine prior to cooling is indicated by a weak negative Eu anomaly (Fig. 10) and by the slightly lower abundances of $\mathrm{Al}_{2} \mathrm{O}_{3}$ (14.77 wt. \%) and Sr (100 ppm).

Cooling Units 12 through 8 seem to be genetically related to each other if $\mathrm{Mg}$ values, $\mathrm{TiO}_{2}, \mathrm{Zr}, \mathrm{Y}, \mathrm{Sr}, \mathrm{Ba}$, and $\mathrm{K}_{2} \mathrm{O}$ are used as discriminant chemical indicators. In this respect and from the petrographic core description, the order of cooling could have started with Cooling Unit 10 (Sample 522B-5-2, 46-48 $\mathrm{cm}$ showing the 


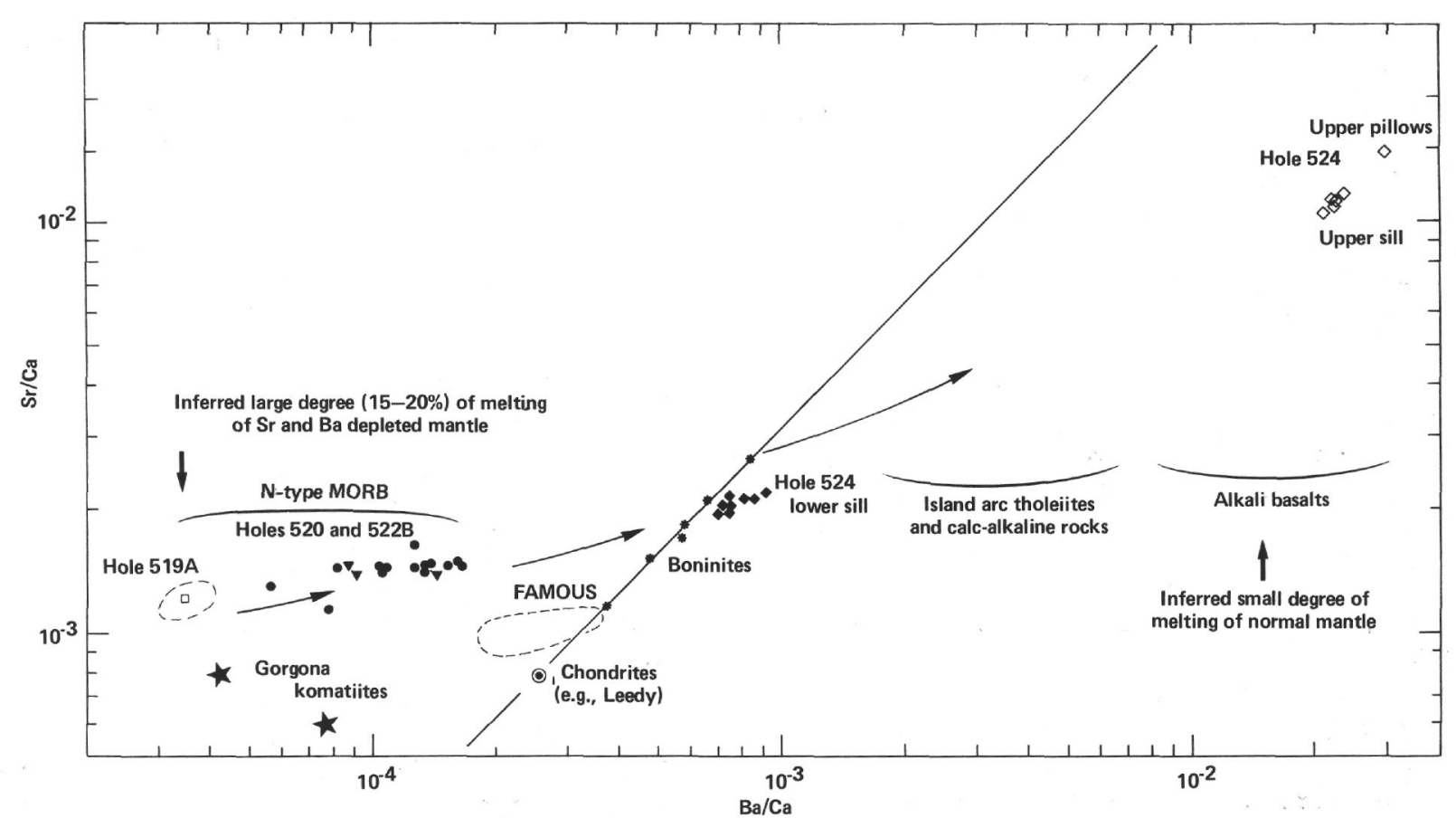

Figure 14. Sr/Ca-Ba/Ca systematics for the Leg 73 basalts (diagram type after Onuma et al., 1981). Sources of data for boninites, Gorgona komatiites, and island arc basalts as in Fig. 12. Data for chondrites from Tera et al. (1970) and Mason (1971); data for FAMOUS basalts from Langmuir et al. (1977). Slightly curved arrows indicate effects of fractional crystallization.

lowest contents of $\mathrm{TiO}_{2}(1.45 \mathrm{wt} . \%), \mathrm{Zr}(90 \mathrm{ppm})$ and $\mathrm{Y}$ (24 ppm); Table 7), followed by Cooling Units 9 and 8, both of the latter being pillows and resting on a glassy chilled top of Cooling Unit 10.

According to the chemical criteria mentioned above, Cooling Unit 12, which is similar in composition to Cooling Unit 10, would be nearly contemporaneous or somewhat later. If later, it would be a sill, which is compatible with its subophitic to intersertal textures. This interpretation also fits with the lack of chilled margins at the lower borders of Cooling Units 10 and 11.

The cooling cycle may have been terminated with the pillow lavas of Cooling Units 9 and 8 . The $\mathrm{TiO}_{2}, \mathrm{Zr}, \mathrm{Y}$, $\mathrm{V}, \mathrm{Ba}$, and $\mathrm{Sr}$ contents of these basalts mark their moderately evolved state. Their contents of $\mathrm{K}_{2} \mathrm{O}(0.29 \mathrm{wt} . \%)$ and $\mathrm{Sc}(46 \mathrm{ppm})$ seem to be enriched compared to typical tholeiites, probably as a result of the alteration of the interstitial glass (Humphris and Thompson, 1978). This is consistent with the high $\mathrm{Fe}_{2} \mathrm{O}_{3} / \mathrm{FeO}$ ratio of 0.96 . Inconsistent with the $\mathrm{Mg}$ value of 0.62 and the higher amounts of the incompatible elements are the higher contents of $\mathrm{Cr}(358 \mathrm{ppm})$ and $\mathrm{Ni}(115 \mathrm{ppm})$ relative to the basalts of Cooling Units 10 and 12. Such a strange composition can only be achieved by the mixture of a relatively primitive melt with a more evolved melt in the upper parts of the magma chamber.

Cooling Units 5 through 7 followed in the eruption sequence. According to their vesicular, intersertal to hyalopilitic textures, their low contents of $\mathrm{K}_{2} \mathrm{O}(0.05-0.09$ wt. \%) and Sc (38-42 ppm), and their low ferric/ferrous ratios, these units can be interpreted as larger lava flows. The major and trace elements are not particularly distinctive and represent the average bulk composition of Hole 522B.
In contrast, the upper four youngest cooling units consist of pillow lava. These rocks are more altered than the underlying flows. The higher contents of $\mathrm{K}_{2} \mathrm{O}(0.25-$ 0.37 wt. \%) and $\mathrm{Sc}(44-49 \mathrm{ppm})$ as well as the $\mathrm{Fe}_{2} \mathrm{O}_{3}$ / $\mathrm{FeO}$ ratios of 0.7 to 0.9 are probably due to the devitrification of interstitial glass.

In terms of differentiation, Cooling Unit 4 represents the least evolved composition $(\mathrm{Mg}$ value $=0.65$ and $\mathrm{Ni}=102 \mathrm{ppm}$ ) and Cooling Unit 3 (Sample 522B-3-3, $116-120 \mathrm{~cm})$ the most differentiated composition $(\mathrm{Mg}$ value $=0.60$ and $\mathrm{Ni}=64 \mathrm{ppm}$ ). The latter basalt has suffered plagioclase fractionation before cooling, as indicated by its relatively low content of $\mathrm{Al}_{2} \mathrm{O}_{3}$ (14.8-14.9 wt. \%). The uppermost pillow basalts (Cooling Units 1 and 2), like Cooling Units 5, 6, 10, and 12, have the average composition of Hole 522B.

In order to evaluate the MORB characteristics, traceelement ratios such as $\mathrm{Ti} / \mathrm{V}$ (Fig. 11), $\mathrm{Zr} / \mathrm{Y}$ (Fig. 12), $\mathrm{Ba} / \mathrm{Zr}$ (Fig. 13), $\mathrm{Ce} / \mathrm{Zr}, \mathrm{Ba} / \mathrm{La}$, and $(\mathrm{Ce} / \mathrm{Sm})_{\mathrm{N}}(\mathrm{Table}$ 3 ), as well as the $\mathrm{Sr} / \mathrm{Ca}-\mathrm{Ba} / \mathrm{Ca}$-systematics (Fig. 14) and distribution are used for discussion.

All the Hole 522B basalts are depleted in the morehygromagmatophile elements $\mathrm{K}, \mathrm{Rb}, \mathrm{Ba}, \mathrm{Nb}$ (Table 7) and LREE (Table 3 ) relative to chondrites, like the basalts of Holes 519A and 520. The Hole 522B basalts can be divided into two major groups: (1) Cooling Units 1 through 12 and (2) Cooling Unit 13. In general, both groups are more differentiated relative to N-type MORBs than those of Holes 519A and 520 due to the fractionation of olivine, spinel, and plagioclase, which yielded higher abundances of $\mathrm{TiO}_{2}$ (Figs. 8A, 9A, and 11), $\mathrm{Zr}$ (Figs. $8 \mathrm{C}$ and 12), Y (Figs. $8 \mathrm{C}$ and 12 ), Ba (Figs. $8 \mathrm{C}$ and 14), and REE (Fig. 10). The lowermost basalt of Cooling Unit 13 has the most primitive composition. From the 
$\mathrm{Y} / \mathrm{Zr}$ and $\mathrm{Ba} / \mathrm{Zr}$ diagrams (Figs. 12 and 13 ) as well as from the $\mathrm{Ce} / \mathrm{Yb}, \mathrm{Ce} / \mathrm{Zr}$, and $\mathrm{Ba} / \mathrm{La}$ ratios (Table 3) it becomes apparent that the Hole 522B basalts have been derived from a different primary magma than those of Holes 519A and 520, a magma slightly enriched in the more-HYG elements. This may have been achieved either by the alternation of different degrees of partial melting or by continuous melting, as proposed by Langmuir et al. (1977). The heterogeneity of the trace-element distribution within the 13 cooling units over a rather limited vertical distance of $19 \mathrm{~m}$, which we interpret as the result of the mixture of melts at different stages of fractionation, seems to be a good argument for the existence of continuous melting processes.

\section{Conclusions}

A sequence of 13 multiple basalt cooling units (19 m of pillow lava and larger flows) were penetrated in Hole 522B. In contrast to the basalts in Holes 519A and 520, the basalts of Hole 522B can be divided into two major groups of tholeiites: (1) Cooling Units 1 through 12 and (2) Cooling Unit 13. The lowermost basalts of Cooling Unit 13 have the most primitive N-type MORB composition and comprise a compositional group distinct from the more evolved MORBs in the twelve units above it.

Major- and trace- (including rare-earth-) element abundances indicate that low-pressure fractionation of olivine, spinel, and plagioclase prior to eruption and quenching are responsible for the compositional spectrum of these basalts.

Incompatible or hygromagmatophile element abundances suggest a chemically homogeneous source region that is slightly more enriched in the more-HYG elements than the source region for Holes 519A and 520. This enrichment may have been caused either by the alternation of different degrees of partial melting or a continuous melting process of a depleted lherzolitic mantle source.

\section{Hole 524}

Hole 524 was drilled on the south flank of the Walvis Ridge (Fig. 1) and thus provided samples from a more complex part of the seafloor than did the other holes. The forces at work at the site should include oceanic ridge volcanism as well as whatever type of activity has given rise to alkalic central eruptions like those in the vicinity of the Walvis Ridge. The first samples from this area were acquired by dredging (Hekinian, 1972; and Humphris and Thompson, 1981); later samples were acquired through the drilling of Hole 359 of Leg 39 of the Deep Sea Drilling Project (Fodor et al., 1977) and through geological and geochemical investigations in the Gough and Tristan da Cunha islands (Smith, 1930; Dunne, 1941; Baker et al., 1964; and O'Nions and Pankhurst, 1974).

The results from Hole 524 do provide evidence of volcanism; three different basaltic rock suites interlayered with volcanic detrital sediments were encountered. A 50- to 60- cm-thick layer of alkaline pillow lava (comprising three cooling units) within the sediments represents the youngest magmatic activity at this part of the ridge segment. Underneath the pillow units appears a 16-m-thick diabase sill of similar (alkaline) composition. The third and lowest rock suite consists of another basaltic sill approximately $9 \mathrm{~m}$ thick. Its composition is tholeiitic (Tables 9 and 10), contrasting strongly with the alkaline rock suites above.

Four volcanic detrital sedimentary layers have been analyzed (Table 11) in order to bracket the compositions of the alkaline rocks within Hole 524. It is apparent from the major- and trace-element chemical composition of these layers that the alkaline volcanic activity began with a sharp boundary above the uppermost tholeiitic basalts, which appear in Core 32, Section 2 (Fig. 4). The volcanic detrital sediments are highly enriched in $\mathrm{K}_{2} \mathrm{O}$ (2.67-5.41 wt. \%), Rb (52 ppm), Ba (240 ppm), Nb (78 ppm), and LREE, but they are very low in $\mathrm{MgO}$ (1.7-3.19 wt.\%), $\mathrm{Cr}(20 \mathrm{ppm})$ and $\mathrm{Ni}(11 \mathrm{ppm})$.

The lowermost rocks encountered in Hole 524 underneath the tholeiitic sill are also composed of volcanic detritus. Their chemical characteristics (e.g., $\mathrm{MgO}(4.92$ wt. \%); $\mathrm{K}_{2} \mathrm{O}(0.92$ wt.\%); and especially $\mathrm{Rb}$ (11 ppm); $\mathrm{Ba}$ (235 ppm); Nb (28 ppm); Cr (122 ppm); Ni (50 ppm); and $\mathrm{Sc}(22 \mathrm{ppm}))$ suggest that the rocks consist mainly of tholeiitic, altered hyaloclastic material cemented by calcareous fossiliferous mud.

To permit an uninterupted discussion of the chemical characteristics of the basalts from Holes 519A, 520, and 522B, the lower tholeiitic diabase sill in Hole 524 will be discussed first.

\section{Lower Sill}

According to the drill records, the lower sill is of multiple origin; it contains three cooling units, which appear to have formed during two injectional phases. The central portion of the sill shows chilled borders at its upper and lower surfaces. The upper and lower portions of the sill are coarse grained up to the contacts with the central portion and show evidence of recrystallization at the contacts (see Hole 524 chapter). As can be seen from the chemical variation of the major elements (Table 9 and Figs. 8A and 9A) and the normative composition (Table 10 and Fig. 5), the upper and lower parts of the sill show a continuation in composition, whereas the composition of the central unit is distinctly different. In contrast to the basalts in Holes 519A, 520, and 522 , the basaltic rocks of the lower sill in Hole 524 are olivine normative (7-12 wt.\% olivine in the CIPW norm) and fall in the central part of the MORB field in the basaltic tetrahedron (Fig. 5). Thus they can be classified as olivine tholeiites (Fig. 6). Using less- and more-HYG elements as chemical discriminators, the diabases possess fairly evolved MORB characteristics: $\mathrm{TiO}_{2}=1.82$ to $1.92 \mathrm{wt} . \%, \mathrm{Y}=29$ to $35 \mathrm{ppm}, \mathrm{Zr}=127$ to $145 \mathrm{pm}, \mathrm{V}=421$ to $471 \mathrm{ppm}$, and $\mathrm{Sc}=38$ to $50 \mathrm{ppm}$. In addition, $\mathrm{K}_{2} \mathrm{O}=0.13$ to 0.23 wt. $\%, \mathrm{Cr}=210$ to 222 $\mathrm{ppm}$, and $\mathrm{Ni}=66$ to $77 \mathrm{ppm}$. Compared with the basalts of Holes 519A, 520, and 522B, these diabases have significantly higher abundances of $\mathrm{Ba}(59-72 \mathrm{ppm}), \mathrm{Sr}$ (162-180 ppm), and the LREE (Table 3). Despite a higher degree of differentiation, the REE pattern of the 
diabases (Fig. 10) does not indicate plagioclase fractionation, as shown by Figure 9A. There is a slight decrease of the HREE relative to N-type MORB patterns.

Plots of $\mathrm{CaO}$ versus $\mathrm{Al}_{2} \mathrm{O}_{3}$ (Fig. 15B) as well as $\mathrm{Al}_{2} \mathrm{O}_{3}$ against the $\mathrm{Mg}$ values (Fig. 15A) show a distinct trend from the top to the bottom of the diabase sill, with $\mathrm{Mg}$ values increasing downward. The central unit analyses fall outside this trend when viewed in the three dimensions (view of Figs. 15A and B orthogonal to each other). The pattern suggests crystal settling or floating in the sill after emplacement, while the parent magma in the chamber from which the first pulse of intrusion came was dif- ferentiating in some other fashion, moving the central unit magma to a slightly different composition from that of the first pulse. As was the case in Hole 519A, crystal fractionation by gravity suggested by the bulk chemistry is not seen in the modal analyses (Carman et al., this vol.), which may simply not be sensitive enough to detect variations as subtle as this.

The $\mathrm{Zr} / \mathrm{Y}$ ratios of the diabases (Table 9, Fig. 12) are of the same magnitude as N-type MORBs in Holes 519A through 522B. However, because the ratios of $\mathrm{Ba} / \mathrm{Zr}$ (Table 9), $\mathrm{Ba} / \mathrm{La}, \mathrm{Ce} / \mathrm{Zr}$, and $\mathrm{Ce} / \mathrm{Yb}$ (Table 3) are distinctly higher, an origin from a weakly depleted mantle
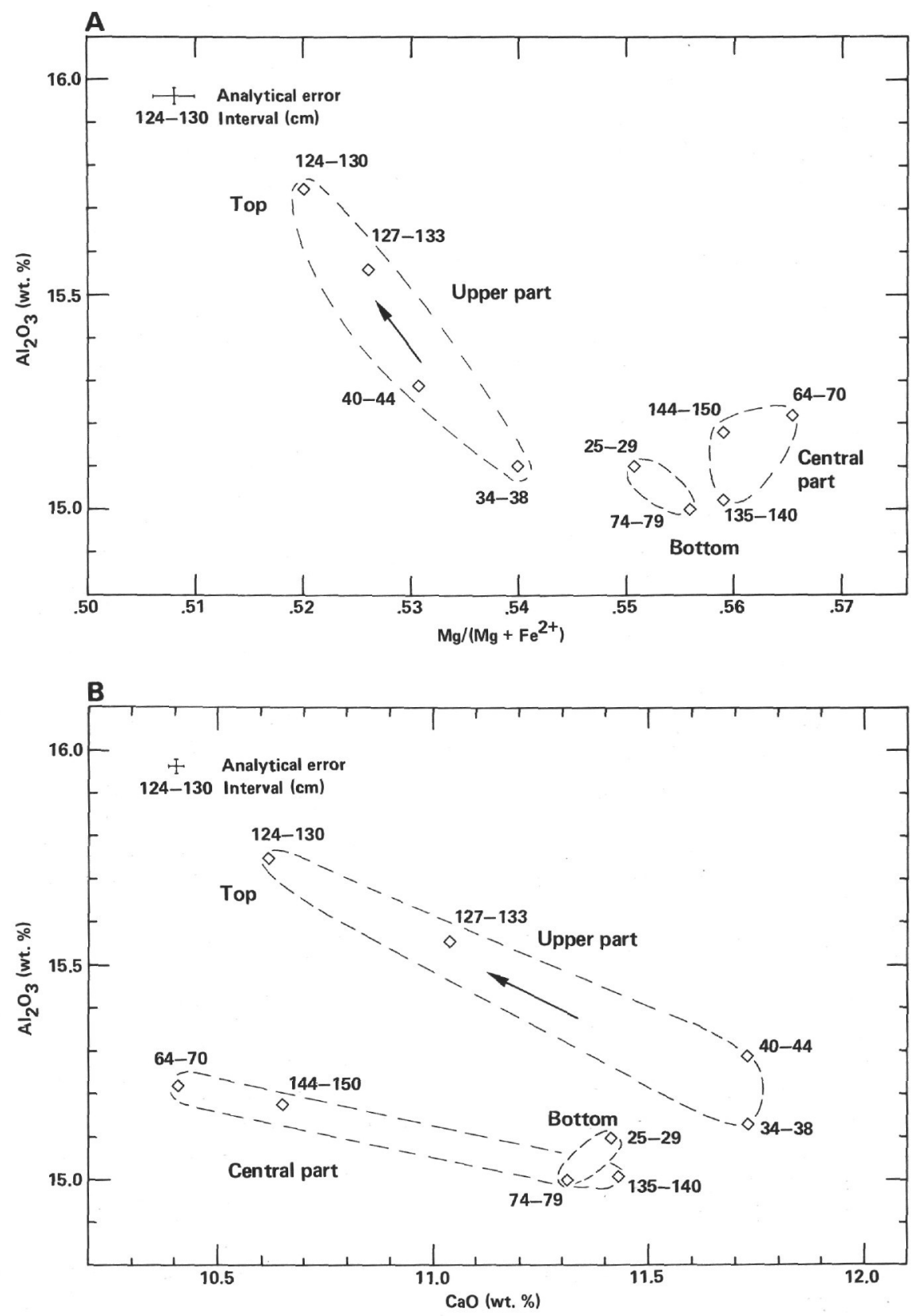

Figure 15. Enlarged $\mathrm{Al}_{2} \mathrm{O}_{3}$ versus $\mathrm{Mg} /\left(\mathrm{Mg}+\mathrm{Fe}^{2+}\right)$ (A) and $\mathrm{Al}_{2} \mathrm{O}_{3}$ versus $\mathrm{CaO}$ (B) variation diagrams of tholeiitic diabases from the lower sill in Hole 524 (Cores 32-34). Arrow indicates the trend of differentiation after emplacement. All values recalculated on a calciteand water-free basis. 
source underneath the Mid-Atlantic Ridge is indicated. The slightly higher alkalinity and the enrichment of the more-HYG elements similar to the transitional (T-type) MORBs of Wood, Joron, et al. (1979) could be a result of mixing with melts generated from greater depth in the upper mantle. The involvement of alkaline melts produced underneath the Walvis Ridge, which passes near the position of Hole 524, is a possibility. In this respect the diabases of the lower sill of Hole 524 resemble basaltic compositions found in the Reykjanes Ridge by Schilling (1975a), in the Azores mantle blob (Schilling, 1975b), and in the Franco-American Mid-Ocean Undersea Study (FAMOUS) area by Wood, Tarney, et al. (1979).

\section{Upper Sill and Pillow Lava}

The pillow lava and the upper diabase sill in Hole 524 are petrographically and compositionally different from the other tholeiitic basalts described here. The basaltic volcanics and subvolcanics belong to the alkalic rock series (Fig. 7). These rocks are characterized by high abundances of $\mathrm{K}_{2} \mathrm{O}$ (between 2.3 and 3.7 wt. \%), abundances that sometimes exceed $\mathrm{Na}_{2} \mathrm{O}$ contents (Table 12).

All these rocks are olivine and hypersthene normative (Table 13), covering a wide normative compositional range between the olivine-plagioclase-diopside plane (the thermal divide) and the diopside-plagioclase-hypersthene plane (the silica saturation plane) in the basaltic tetrahedron (Fig. 5).

According to a new classification scheme for alkalic rock series (Miyashiro, 1978), the alkalic rocks of Hole 524 (alkali olivine basalts) fit into the straddle Type B category, an alkalic volcanic association which may have been differentiated at pressures above $10 \mathrm{kbar}$ or under some hydrous or oxidizing conditions where the olivineplagioclase-diopside plane is no longer a thermal divide. Miyashiro (1978) assumes that the crystallization of lowsilica minerals such as amphibole, magnetite, and apatite may be the means of deriving such a trend. In fact, large quantities of ilmenite, magnetite, and apatite are present. This also appears in the CIPW norm (Table 13): ilmenite and magnetite constitute up to $10 \mathrm{wt} . \%$ of the rocks and apatite constitutes 2.7 to 5.1 wt. \%. Amphibole occurs sporadically.

The bulk chemical composition of the Hole 524 alkaline basalts is given in Table 12 and shown in Figs. 8A to $\mathrm{C}, 15$, and 16. The high contents of $\mathrm{TiO}_{2}(3.5-4.3$ wt. \%) and $\mathrm{P}_{2} \mathrm{O}_{5}$ (1.1-2.07 wt. \%) as well as of the lessand more-HYG elements $(\mathrm{Ba}=990-1350 \mathrm{ppm} ; \mathrm{Rb}=$ 15-77 ppm; $\mathrm{Sr}=415-672 \mathrm{ppm} ; \mathrm{Nd}=37-42 \mathrm{ppm} ; \mathrm{Y}=$ 35-42 ppm; and $\mathrm{Zr}=255-287 \mathrm{ppm}$ ) and REE (e.g., $\mathrm{Ce}=100-140 \mathrm{ppm}$ ) are indicative of the high alkalinity of these rocks. In contrast, $\mathrm{Cr}(<10-21 \mathrm{ppm})$ and $\mathrm{Ni}$ $(<3-14 \mathrm{ppm})$ contents are very low and indicate a high degree of differentiation.

The more-HYG trace elements such as $\mathrm{Ba}, \mathrm{Rb}$, and $\mathrm{Sr}$ and LREE correlate only vaguely with $\mathrm{K}_{2} \mathrm{O}$ (Table 12). Better correlations exist between $\mathrm{Mg}$ values and $\mathrm{TiO}_{2}, \mathrm{~V}$, and Sc (Figs. 8A and B). The ferric/ferrous ratios, $\mathrm{CO}_{2}$ contents (indicative of calcite), and the relatively high $\mathrm{H}_{2} \mathrm{O}^{+}$content (Table 12) are at least partly due to alteration, which is stronger in the pillow lavas than in the diabases.

The less evolved rocks of the alkalic suites in Hole 524 seem to occur in the middle and lower parts of the upper diabase sill. Sample 524-30-2, 72-76 cm has the highest $\mathrm{Mg}$ value $(0.46)$, the lowest $\mathrm{TiO}_{2}$ content (3.5 wt. \%), and the lowest $\mathrm{V}$ content (195 ppm).

The upper pillow lavas are more differentiated than the diabases, Sample 524-28-3, 64-69 $\mathrm{cm}$ having the lowest $\mathrm{Mg}$ value $(0.33)$ and the highest contents of $\mathrm{TiO}_{2}$ (4.31 wt. \%), V (348 ppm). and Sc (37 ppm) as well as of $\mathrm{P}_{2} \mathrm{O}_{5}\left(2.07\right.$ wt. \%), $\mathrm{K}_{2} \mathrm{O}$ (3.71 wt. \%), Rb (71 ppm) and $\mathrm{Ba}$ (1350 ppm) and high LREE (Table 3 and Fig. 16). Although the $\mathrm{CaO}$ contents are relatively low in the highly differentiated pillows, it seems improbable that only plagioclase fractionation occurred in its parent magma. This is because (1) $\mathrm{Al}_{2} \mathrm{O}_{3}$ content is even greater than in the less fractionated pillow samples (524-28-3, 93-97 cm and 524-28-3, 105-109 cm; Table 12) and (2) the rocks exhibit a slight positive Eu anomaly (Fig. 16). This anomaly reflects the high abundances of alkali feldspar and plagioclase in the rocks.

Clinopyroxene and other low-silica and low-potassic phases such as the Fe-Ti oxides and probably amphibole are considered to be the controlling minerals during fractionation. The low abundances of $\mathrm{Cr}$ and $\mathrm{Ni}$ in all the samples of the alkalic suites in Hole 524 indicate either that olivine and spinel must have fractionated at early stages or that $\mathrm{Cr}$ and $\mathrm{Ni}$ were never concentrated in the primary alkaline melt. The pillows and the sill rocks may have originated from different magma batches, because their trace-element ratios are distinctly different (Table 12 and Figs. 11-14). A genetic relation between pillows and diabases based on fractionation seems to be precluded by the trace-element characteristics (Figs. 8B and $\mathrm{C}$ ).

\section{Nature and Origin of the Walvis Ridge}

Alkali olivine basalts were encountered in the upper part of Hole 524 at the southeastern flank of the Walvis Ridge (Fig. 1). In order to establish genetic relationships between this ridge (a possible chain of alkaline volcanic seamounts) and the alkaline basalts of Hole 524, the alkaline Hole 524 basalts can be compared with the alkaline volcanics of the Tristan da Cunha Islands. This group of volcanic islands, which probably represents the tips of a huge composite volcanic unit rising from the seafloor (which is at a depth of about $3600 \mathrm{~m}$ ), is one of the most isolated pieces of land on the globe and marks the intersection of the Walvis Ridge with the Mid-Atlantic Ridge (precisely on Anomaly 5, $400 \mathrm{~km}$ east of the central ridge) at latitude $37^{\circ} \mathrm{S}$ and longitude $12^{\circ} \mathrm{W}$. The Mid-Atlantic Ridge axis seems to be elevated over the Tristan da Cunha-Gough platform, and the crest is ill defined. Along the MAR crest from $47^{\circ} \mathrm{S}$ to $31^{\circ} \mathrm{S}$ the mid-ocean-ridge basalts show a change in alkalinity, a change indicated by the variation of $\mathrm{La} / \mathrm{Sm}$ from 0.64 to 1.44 (Schilling, 1981).

The predominant rock types on the Tristan da Cunha Islands are alkali olivine basalts and hornblende-bearing alkali basalts. The more differentiated rocks that exist 


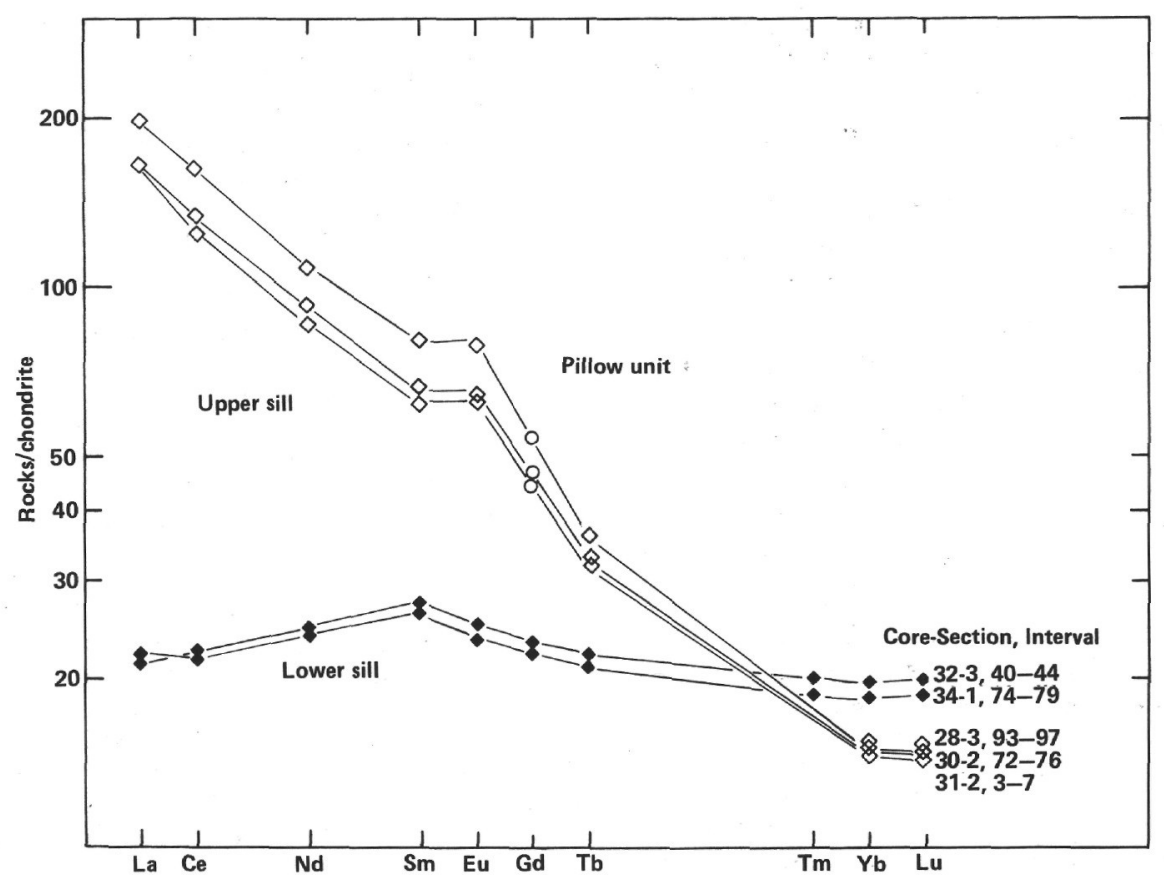

Figure 16. Chondrite-normalized REE plot for the basalts and diabases from Hole 524.

on the islands (straddle Type B alkalic association according to Miyashiro, 1978; e.g., trachybasalts, mugearites, tephritic basalts, trachyandesites, biotite-trachytes, and sodalite-trachytes) were probably formed by the crystal fractionation of plagioclase, clinopyroxene, hornblende, and magnetite (Dunne, 1941; and Baker et al., 1964).

Ash-flow tuffs compositionally similar to the trachytic rocks of the Tristan da Cunha Islands were cored from a seamount at the southwest extremity of the Walvis Ridge (Hole 359, Leg 39; Fig. 1; Fodor et al., 1977). This seamount probably erupted at Eocene time, producing lavas that overlie normal MAR basement.

The olivine basalts and trachybasalts of the Tristan da Cunha Islands are of almost the same composition as the alkaline sill in Hole 524 (Fig. 7). Thus, the two parental magmas may have originated from similar upper mantle sources. In addition, Richardson et al. (1982) showed that incompatible element and $\mathrm{Pb}$-isotopic systematics preclude an extensive involvment of depleted (N-type) MORB or mantle source material in the petrogenesis of the Walvis Ridge alkali basalts.

The question arises as to whether these basalts were derived by the partial melting of upper mantle, a process similar to the processes that generate enriched (E-type) MORBs (Richardson et al., 1982), or by the rise of a lower mantle plume through the upper mantle.

Petrologic evidence (e.g., amphibole fractionation) and isotopic evidence favor the first hypothesis. Additional evidence of a chemically modified upper mantle beneath the Walvis Ridge is provided by regional Rayleigh wave dispersion characteristics (Chave, 1979).

The interpretation of the Walvis Ridge as a line of hot-spot alkaline volcanoes (Morgan, 1972; and Wilson, 1973) might be realistic. However, it is difficult to be- lieve that the hot-spot activity provided energy for plate motion (Morgan, 1972).

On the basis of an echelon structure of the Walvis Ridge (three main ridge segments of different strike), Goslin and Sibuet (1975) proposed a modified hot-spot model in which magmas were able to rise from the upper mantle through transverse zones of weakness.

Ocean-floor spreading in the South Atlantic, which may have started at the beginning of the Cretaceous approximately 140 m.y. ago (LePichon and Hayes, 1971; Phillips and Forsyth, 1972; and Morgan, 1972), could have produced a chain of alkaline volcanoes by moving the oceanic plate (African plate) toward the northeast over a nearly fixed hot spot in the mantle. If the distance of $\sim 1600 \mathrm{~km}$ between Hole 524 and the Tristan da Cunha Islands is divided by the 60- to $65-\mathrm{m} . y$. time interval of volcanic activity, the resulting spreading rate is 2 to $2.5 \mathrm{~cm} / \mathrm{yr}$. This fits the approximate spreading rates derived by the extrapolation of the magnetic anomalies in the South Atlantic (Larson and Pitman, 1972); it also agrees with the rates suggested by previous DSDP records (Maxwell et al., 1970).

\section{Conclusions}

Hole 524 was drilled on the southeastern flank of the Walvis Ridge and thus provided samples of a more complex part of the South Atlantic seafloor. Three different basalt rock suites were encountered (with minor intercalated volcanic detrital sediments). These include, from top to bottom, an alkali basaltic pillow lava, a 16-mthick alkaline diabase sill with an age of $\sim 65 \mathrm{~m} . \mathrm{y}$., and a second sill that is approximately $9 \mathrm{~m}$ thick, $\sim 74 \mathrm{~m}$.y. old, and tholeiitic in composition. The incompatible element abundances as well as the $\mathrm{Ti}-\mathrm{V}-\mathrm{Zr}-\mathrm{Ba}-\mathrm{Y}-\mathrm{La}-\mathrm{Ce}-$ $\mathrm{Yb}$ interelement relationships show that the tholeiitic 
basalts are generally N-type MORBs. Their slightly higher alkalinity and enrichment in the more-HYG elements (characteristics similar to T-type MORBs) could be a result of mixing with melts generated from greater depth in the upper mantle.

The chemical characteristics of the alkali basalts of Hole 524 are very similar to the characteristics of the basaltic lavas of the Walvis Ridge in Holes 525A, 527, and 528 (Leg 74) and the Tristan da Cunha group volcanoes, which are located at the intersection of the Walvis Ridge with the Mid-Atlantic Ridge, about $400 \mathrm{~km}$ east of the Mid-Atlantic Ridge crest. Thus, the Walvis Ridge may plausibly be interpreted as a line of hot-spot alkaline volcanoes that has been generated since the opening of the South Atlantic during the Cretaceous. Petrologic, chemical, and isotopic evidence suggest that the basalts were generated by the partial melting of upper mantle material underneath the Walvis Ridge rather than by the rise of a lower mantle plume through the upper mantle.

\section{ACKNOWLEDGMENTS}

The XRF analyses were made with the assistance of A. Esenwein from the Eidgenössische Materialprüfungsanstalt (EMPA), Dübendorf, Switzerland. Neutron activation analyses of the rare earth elements were carried out with the help of S. Bajo at the Eidgenössisches Institut für Reaktorforschung (EIR), Würenlingen, Switzerland.

We also thank E. A. Mathez (in Seattle) and S. Hurley (in Zürich) for thorough reviews that improved the manuscript.

\section{REFERENCES}

Bajo, S., and Wyttenbach, A., 1980. Extraction liquide-liquide des terres rares et application à leur dosage dans le silicates par activation neutronique. EIR Technische Mitteilung (internal report), TM-44-80-2.

Baker, P. E., Gass, I. G., Harris, P. G., and LeMaitre, R. W., 1964. The volcanological report of the Royal Society expedition to Tristan da Cunha, 1962. Philos. Trans. R. Soc. London Ser. A, 256:439-575.

Chave, A. D., 1979. Lithospheric structure of the Walvis Ridge from Rayleigh wave dispersion. J. Geophys. Res., 84:6840-6848.

Dalrymple, G. B., and Lanphere, M. A., 1969. Potassium-Argon Dating: San Francisco (W. H. Freeman).

Dietrich, V., Emmermann, R., Oberhaensli, R., and Puchelt, H., 1978. Geochemistry of basaltic and gabbroic rocks from the West Mariana basin and the Mariana trench. Earth Planet. Sci. Lett., 39:127-144.

Dietrich, V., Gansser, A., Sommerauer, J., and Cameron, W. E., 1981. Palaeogene komatiites from Gorgona island, East Pacifica primary magma for ocean floor basalts. Geochem J., 15:141-161.

Dietrich, V., Oberhaensli, R., and Walpen, P., 1976. Roentgenfluoreszenzanalyse der Silikatgesteine (internal report): Zürich (Institut fur Kristallographie und Petrographie der ETH-Zürich).

Dunne, J. C., 1941. Volcanology of the Tristan da Cunha Group: Results of the Norwegian Scientific Expedition to Tristan da Cunha 1937-1938, No. 2: Oslo (Det Norske Videnskap-Akademi i Oslo).

Floyd, P. A., and Tarney, J. 1979. First-order alteration chemistry of Leg 49 basement rocks. In Luyendyk, B. P., Cann, J. R., et al., Init. Repts. DSDP, 49: Washington (U.S. Govt. Printing Office), 693-708.

Fodor, R. V., Keil, Husler, J. W., and McKee, E. H., 1977. Petrology and $\mathrm{K}-\mathrm{Ar}$ age of volcanic tuff and ash from the Walvis seamount province, DSDP Site 359, Leg. 39. In Supko, P. R., PerchNielsen, K., et al., Init. Repts. DSDP, 39: Washington (U.S. Govt. Printing Office), 525-536.

Frey, F. A., Bryan, W. B., and Thompson, G., 1974. Atlantic Ocean floor: geochemistry and petrology of basalts from Legs 2 and 3 of the Deep-Sea Drilling Project. J. Geophys. Res., 79:5507-5527.

Gill, J., 1981. Orogenic Andesites and Plate Tectonics: Berlin (Springer-Verlag).
Goslin, J., and Sibuet, J.-C., 1975. Geophysical study of the easternmost Walvis Ridge, South Atlantic: deep structure. Geol. Soc. Am. Bull., 86:1713-1724.

Haskin, L. A., Haskin, M. A., Frey, F. A., and Wildeman, T. R., 1968. Relative and absolute terrestrial abundances of rare earths. In Ahrens, L. A. (Ed.), Origin and Distribution of the Elements, 30:889-912.

Hekinian, R., 1972. Volcanics from the Walvis Ridge. Nature, 239: 91-93.

Humphris, S. E., and Thompson, G., 1978. Trace element mobility during hydrothermal alteration of oceanic basalts. Geochim. Cosmochim. Acta, 42:127-136.

1981. Petrological and geochemical studies of rocks dredged from the Walvis Ridge, South Atlantic. Trans. Am. Geophys. Union, 62:424.

Ingamells, C. O., 1970. Lithium metaborate flux in silicate analysis, Anal. Chim. Acta, 52(2):323-334.

Jagoutz, E., Palme, H., Baddenhausen, H., Blum, K., Cendales, M., Dreibus, G., Spettel, B., Lorenz, V., and Waenke, H., 1979. The abundances of major, minor and trace elements in the Earth's mantle as derived from primitive ultramafic nodules. Proc. Tenth Lunar Planet Sci. Conf., pp. 2031-2050.

Langmuir, C. H., Bender, J. F., Bence, A. E., Hanson, G. N., and Taylor, S. R., 1977. Petrogenesis of basalts from the FAMOUS area: Mid-Atlantic Ridge. Earth Planet. Sci. Lett., 36:133-156.

Larson, R. L., and Pitman, W. C., III, 1972. World-wide correlation of Mesozoic magnetic anomalies, and its implications. Geol. Soc. Am. Bull., 83:3645-3662.

LePichon, X., and Hayes, D. E., 1971. Marginal offsets, fracture zones, and the early opening of the South Atlantic. J. Geophys. Res., 76:6283-6293.

McDonald, G. A., and Katsura, T., 1964. Chemical composition of Hawaiian lavas. J. Petrol., 5:82-133.

Mason, B., 1971. Handbook of Elemental Abundances in Meteorites: New York (Gordon and Breach Science Publ.).

Masuda, A., Nakamura, N., and Tanaka, T., 1973. Fine structures of mutually normalized rare-earth patterns of chondrites. Geochim. Cosmochim. Acta, 37:239-248.

Maxwell, A. E., Von Herzen, R. P., et al., Init. Repts. DSDP, 3: Washington (U.S. Govt. Printing Office).

Miyashiro, A., 1978. Nature of alkalic volcanic rock series. Contrib. Mineral. Petrol., 66:91-104.

Morgan, W. J., 1972. Deep mantle convection plumes and plate motions. Am. Assoc. Pet. Geol. Bull., 56:203-213.

Nisbet, E. G., Dietrich, V. J., and Esenwein, A., 1979. Routine trace element determination in silicate minerals and rocks by X-ray fluorescence. Fortschr. Mineral., 57:264-279.

O'Nions, R. K., Evensen, N. M., Hamilton, P. J., and Carter, S. R., 1978. Melting of the mantle past and present: isotope and trace element evidence. Philos. Trans. $R$. Soc. London Ser. A, 288: 547-559.

O'Nions, R. K., and Pankhurst, R. J., 1974. Petrogenetic significance of isotope and trace element variations in volcanic rocks from the Mid-Atlantic. J. Petrol., 15:603-634.

Onuma, N., Hirano, M., and Isshiki, N., 1981. Sr/Ca-Ba/Ca systematics in four volcanoes of Oshima, Izu Islands, Japan. Geochem. J., 15:315-324.

Pearce, J., and Cann, J. R., 1973. Tectonic setting of basic volcanic rocks determined using trace element analysis. Earth Planet. Sci. Lett., 19:290-300.

Pearce, J., and Norry, M. J., 1979. Petrogenetic implications of Ti, $\mathrm{Zr}, \mathrm{Y}$ and $\mathrm{Nb}$ variations in volcanic rocks. Contrib. Mineral. Petrol., 69:33-47.

Phillips, J. D., and Forsyth, D., 1972. Plate tectonics, paleomagnetism, and the opening of the Atlantic. Geol. Soc. Am. Bull., 83: 1579-1600.

Richardson, S. H., Erlank, A. J., Duncan, A. R., and Reid, D. L., 1982. Correlated $\mathrm{Nd}, \mathrm{Sr}$ and $\mathrm{Pb}$ isotope variation in Walvis Ridge basalts and implications for the evolution of their mantle source. Earth Planet. Sci. Lett., 59:327-342.

Richardson, S. H., Erlank, A. J., Reid, D. L., and Duncan, A. R., in press. Major and trace element and $\mathrm{Nd}$ and $\mathrm{Sr}$ isotope geochemistry of basalts from DSDP Leg 74 Walvis Ridge transect. In Moore, T. C., Jr., Rabinowitz, P. D., et al., Init. Repts. DSDP, 74: Washington (U.S. Govt. Printing Office). 
Saunders, A. D., Tarney, J., Marsh, N. G., and Wood, D. A., 1980. Ophiolites as ocean crust or marginal basin crust: a geochemical approach. Proc. Int. Ophiolite Symp. Cyprus 1979, pp. 193-204.

Schilling, J. G., 1971. Sea-floor evolution: rare earth evidence. Philos. Trans. R. Soc. London Ser. A, 268:663-706.

1973. Iceland mantle plume: geochemical study of Reykjanes Ridge. Nature, 242:565-575.

1975a. Rare earth variations across "normal segments" of the Reykjanes Ridge, 600-530 N, Mid-Atlantic Ridge, 290 S, and East Pacific Rise 20-190 S, and evidence on the composition of the underlying low velocity layer. J. Geophys. Res., 80:1459-1473.

1975b. Azores mantle blob: rare earth evidence. Earth Planet. Sci. Lett., 25:103-115.

Schilling, J. G., Humphris, S. E., and Thompson, G., 1981. Tristan da Cunha hotspot. Trans. Am. Geophys. Union, 62:424.

Schubiger, P. A., Patry, J., and Wyttenbach, A., 1978. Jane-Ein Vielzweckprogramm zur quantitativen und qualitativen Auswertung von Gammaspektren. EIR-Bericht (internal report), 345: Würenlingen, Switzerland (Eidgenössiches Reaktorinstitut).

Shervais, J. W., 1982. Ti-V plots and the petrogenesis of modern and ophiolitic lavas. Earth Planet. Sci. Lett., 59:101-118.

Shido, F. A., Miyashiro, A., and Ewing, M., 1971. Crystallization of abyssal tholeites. Contrib. Mineral. Petrol., 31:251-266.

Sixta, V., 1977. Coulometric determinations of carbonates in rock samples. Z. Anal. Chem., 285:369-372.

Smith, W. C., 1930. Petrography of the Tristan da Cunha Group. Report of the Geological Collections Made during the Voyage of the Quest on the Shackleton-Rowett Expedition to the South Atlantic and Weddell Sea in 1921-1922: London (British Museum Nat. History), pp. 72-87.

Staudigel, H., Hart, S. R., and Richardson, S. H., 1981. Alteration of the oceanic crust: process and timing. Earth Planet. Sci. Lett., 52:311-327.

Sun, S. S., Nesbitt, R. W., and Sharaskin, A. Y., 1979. Geochemical characteristics of Mid-Ocean-Ridge-Basalts. Earth Planet. Sci. Lett., 44:119-138.

Tarney, J., Saunders, A. D., Weaver, S. D., Donnellan, N. C. B., and Hendry, G. L., 1979. Minor-element geochemistry of basalts from Leg 49, North Atlantic Ocean. In Luyendyk, B. P., Cann, J. R., et al., Init. Repts. DSDP, 49: Washington (U.S. Govt. Printing Office), 657-692.

Taylor, S. R., and Gorton, M. P., 1977. Geochemical application of spark source mass spectrography. III. Element sensitivity, precision and accuracy. Geochim. Cosmochim. Acta, 41:1375-1380.

Tera, F., Eugster, O., Burnett, D. S., and Wasserburg, G. J., 1970. Comparative study of $\mathrm{Li}, \mathrm{Na}, \mathrm{K}, \mathrm{Rb}, \mathrm{Cs}, \mathrm{Ca}, \mathrm{Sr}, \mathrm{Ba}$ abundances in achondrites and in Apollo 11 lunar samples. Proc. Apollo 11 Lunar Sci. Conf., 2:1637-1657.

Thompson, G., Shido, F., and Miyashiro, A., 1972. Trace element distribution in fractionated oceanic basalts. Chem. Geol., 9:89-97.

Thornton, C. P., and Tuttle, O. F., 1960. Chemistry of igneous rocks. I. Differentiation index. Am. J. Sci., 258:664-684.

Treuil, M., and Varet, J., 1973. Critères volcanologiques, pétrologiques et géochimiques de la genese et de la differenciation des magmas basaltiques: example de l'Afar. Bull. Soc. Géol. Fr., 15: 506-540.

White, W. M., and Schilling, J.-G., 1978. The nature and origin of geochemical variations in Mid-Atlantic Ridge basalts from the central North-Atlantic. Geochim. Cosmochim. Acta, 42:1501-1516.

Wilson, J. T., 1973. Mantle plumes and plate motions. In Irving, E. (Ed.), Mechanisms of Plate Tectonics. Tectonophysics, 19:149-164.

Wood, D. A., Joron, J. L., Marsh, N. G., Tarney, J., and Treuil, M., 1980. Major- and trace-element variations in basalts from the North Philippine Sea drilled during Deep Sea Drilling Project Leg 58: a comparative study of back-arc-basin basalts with lava series from Japan and mid-ocean ridges. In Klein, G. DeV., Kobayashi, K., et al., Init. Repts. DSDP, 58: Washington (U.S. Govt. Printing Office), 873-894.

Wood, D. A., Joron, J. L., and Treuil, M., 1979. A re-appraisal of the use of trace elements to classify and discriminate between magma series and different tectonic settings. Earth Planet. Sci. Lett., 45:326-336.

Wood, D. A., Tarney, J., Varet, J., Saunders, A. D., Bougault, H., Joron, J. L., Treuil, M., and Cann, J. R., 1979. Geochemistry of basalts drilled in the North Atlantic by IPOD Leg 49: implications for mantle heterogeneity. Earth Planet. Sci. Lett., 42:77-97.

Date of Initial Receipt: June 17, 1982 